\title{
Diode-pumped single-frequency-Nd:YGG-MOPA for water-vapor DIAL measurements: design, setup and performance
}

\author{
J. Löhring • A. Meissner · D. Hoffmann • A. Fix • \\ G. Ehret • M. Alpers
}

Received: 17 May 2010 / Revised version: 25 September 2010 / Published online: 23 December 2010

(C) The Author(s) 2010. This article is published with open access at Springerlink.com

\begin{abstract}
A diode-pumped Q-switched and injection-seeded single-frequency laser, generating tunable laser radiation at $935 \mathrm{~nm}$, is presented. Using $\mathrm{Nd}$ : $\mathrm{YGG}\left(\mathrm{Y}_{3} \mathrm{Ga}_{5} \mathrm{O}_{12}\right)$ as the active medium, the laser that was developed to serve as a transmitter for water-vapor lidar measurements. The configuration consists of a stable resonator in rod geometry that is injection seeded by a narrowband diode laser and stabilized by the ramp-and-fire technique. Energy scaling was done in a power amplifier in slab geometry. Both oscillator and amplifier crystal were diode pumped at $806 \mathrm{~nm}$. More than $30 \mathrm{~mJ}$ pulse energy at $100 \mathrm{~Hz}$ repetition rate with a beam propagation factor of $\mathrm{M}^{2}<1.4$ and pulse duration of $52 \mathrm{~ns}$ in single-frequency mode were generated. To our knowledge this is the first direct generation of $935 \mathrm{~nm}$ Q-switched pulses from Nd:YGG suitable for water-vapor measurements. The reported results show great promise of this laser in applications where high efficiency and reduced complexity is indispensable, such as for spaceborne or airborne water-vapor lidar instruments.
\end{abstract}

J. Löhring $(\bowtie)$ · A. Meissner · D. Hoffmann

Fraunhofer-Institut für Lasertechnik (ILT), Steinbachstr. 15, 52074 Aachen, Germany

e-mail: jens.loehring@ilt.fraunhofer.de

A. Fix · G. Ehret

Institut für Physik der Atmosphäre, Deutsches Zentrum für Luftund Raumfahrt (DLR), 82234 Oberpfaffenhofen, Germany

\section{Alpers}

Raumfahrt-Agentur, Erdbeobachtung, Bonn-Oberkassel, Deutsches Zentrum für Luft- und Raumfahrt (DLR), Königswinterer Straße 522-524, 53227 Bonn, Germany

\section{Introduction}

Many applications in science and technology require specific laser sources with optimized performance at certain wavelengths not readily accessible using common lasers [1]. One such example is active remote sensing of water vapor using the differential absorption lidar (DIAL) technique [2].

This technique enables highly accurate measurements of the earth's most important greenhouse gas [3] from the ground to the lower stratosphere. The knowledge of vertically resolved water-vapor profiles is a prerequisite for numerical weather prediction and climate research since atmospheric humidity controls weather and climate on all temporal and spatial scales. The operation of a DIAL system on board satellites is of particular interest since this enables global measurements to be made which exceed the capabilities of current observation systems like radio-sondes or passive satellite sensors [4]. Therefore, in the past several years several missions have been proposed to measure water-vapor profiles from space [5-9].

An appropriate laser source for spaceborne $\mathrm{H}_{2} \mathrm{O}$-DIAL requires a pulsed laser with pulse durations in the sub 200-ns range with demanding requirements concerning spectral and spatial properties as well as high pulse energy and average power and an emission wavelength that can be tuned to the specific absorption lines in the $935-\mathrm{nm}$ band of water vapor $[5,9]$.

It is obvious that the operation of a lidar transmitter onboard a satellite requires highly efficient diode-pumped all-solid-state sources. For this purpose either Ti:Sapphire lasers [5] or optical parametric oscillators (OPOs) have been suggested in most cases [6,9]. Those sources are pumped, making use of the second harmonic of e.g. Nd:YAG laser at $1064 \mathrm{~nm}$. Due to the frequency conversion process these lasers are, however, inefficient. In principle, it should be pos- 
sible to come up with more efficient and less complex allsolid-state lasers that directly generate light in the respective wavelength range.

It has been suggested that Nd-doped $\mathrm{Y}_{3} \mathrm{Ga}_{x} \mathrm{Al}_{5-x} \mathrm{O}_{12}$ (YGAG) lasers can be specifically tuned within the required wavelength range at $935 \mathrm{~nm}$ by changing the material composition $x$ with $(0 \leq x \leq 5)$ and thus the lattice parameters $[10,11]$.

In addition, a few experimental studies with this and other innovative laser materials were performed trying to address wavelengths in the respective wavelength range.

Walsh et al. studied different Nd-doped composite laser materials $\quad\left(\mathrm{YAG}_{x} \mathrm{YGG}_{1-x}, \quad \mathrm{YAG}_{x} \mathrm{YSGG}_{1-x}, \mathrm{YAG}_{x}\right.$ $\mathrm{YSAG}_{1-x}, \quad \mathrm{YAG}_{x} \mathrm{GSAG}_{1-x}, \quad \mathrm{YAG}_{x} \mathrm{GGG}_{1-x}, \mathrm{YSAG}_{x^{-}}$ $\mathrm{GGG}_{1-x}, \mathrm{YAG}_{x}\left[\mathrm{YSAG}_{y} \mathrm{GGG}_{1-y}\right]_{1-x}$ ) for efficient laser operation around $940 \mathrm{~nm}$ [12]. Here, $x$ and $y$ with $(0 \leq$ $x \leq 1)$ and $(0 \leq y \leq 1)$ describe mixing ratios of different garnets.

Different Nd-doped garnets ( $\mathrm{Y}_{3} \mathrm{Ga}_{5} \mathrm{O}_{12}$ (YGG), $\mathrm{Lu}_{3} \mathrm{Ga}_{5}$ $\left.\mathrm{O}_{12}(\mathrm{LuGG})\right)$ and mixed garnets $\left(\mathrm{Y}_{3}(\mathrm{Sc}, \mathrm{Al})_{5} \mathrm{O}_{12}\right.$ (YSAG), $\mathrm{La}_{3}(\mathrm{Lu}, \mathrm{Ga})_{5} \mathrm{O}_{12} \quad(\mathrm{LaLuGG}), \mathrm{Gd}_{3}(\mathrm{Sc}, \mathrm{Ga})_{5} \mathrm{O}_{12}$ (GSGG), $\mathrm{Gd}_{3}(\mathrm{Sc}, \mathrm{Al})_{5} \mathrm{O}_{12}$ (GSAG)) were spectroscopically investigated in order to address the water-vapor absorption lines around $935 \mathrm{~nm}, 942 \mathrm{~nm}$ and $944 \mathrm{~nm}$ [13]. Furthermore, experiments with $\mathrm{Nd}: \mathrm{Gd}_{3} \mathrm{Sc}_{2} \mathrm{Al}_{3} \mathrm{O}_{12}$-crystals were performed to address the wavelength group around $942 \mathrm{~nm}[14,15]$.

The potential of mixed garnet or pure garnet crystals at uncommon transitions for the generation of application specific wavelengths in the relevant parameter regime was demonstrated only in few experiments. $150 \mathrm{~ns}$ Q-switched (QS) pulses at $942 \mathrm{~nm}$ in longitudinal multimode with $25 \mathrm{~mJ}$ pulse energy at a repetition rate of $10 \mathrm{~Hz}$ with an optical to optical efficiency of $9 \%$ were demonstrated in a diode-pumped Nd:GSAG laser using a linear twistedmode cavity [15]. Recently, single-frequency operation of a Q-switched and diode-end-pumped Nd:GSAG ring oscillator with an output energy of $13.2 \mathrm{~mJ}$ at $10 \mathrm{~Hz}$ repetition rate was demonstrated [16]. Walsh et al. demonstrated QS pulses with $100 \mathrm{~mJ}$ pulse energy, $160 \mathrm{~ns}$ pulse duration and $1 \mathrm{~Hz}$ repetition rate at $944 \mathrm{~nm}$, but in a flashlamp-pumped $\mathrm{Nd}: \mathrm{YAG}_{(0.18)} \mathrm{YSAG}_{(0.82)}$ laser [12]. Axenson et al. demonstrated about $75 \mathrm{~mJ}$ QS-pulses at a repetition rate of $1 \mathrm{~Hz}$ at $946 \mathrm{~nm}$ generated by the $\mathrm{R}_{1}-\mathrm{Z}_{5}$-transition in Nd:YAG. They used a folded oscillator setup with three diode-endpumped crystals. The total pump energy was about $910 \mathrm{~mJ}$, which corresponds to an extraction efficiency of $8.2 \%$ [17]. We reported firstly about QS laser operation at $935 \mathrm{~nm}$ in a diode-pumped $\mathrm{Nd}$ :YGG laser where $4 \mathrm{~mJ}$ pulses with a pulse duration of $<60 \mathrm{~ns}$ at a repetition rate of $100 \mathrm{~Hz}$ with an optical-to-optical efficiency of $9 \%$ [27] were generated. $\mathrm{Li}$ et al. demonstrated a diode-pumped continuous wave $(\mathrm{CW}) \mathrm{Nd}$-doped $\mathrm{Ca}_{3}(\mathrm{NbGa})_{2-x} \mathrm{Ga}_{3} \mathrm{O}_{12}$ (Nd:CNGG) laser that generates $1.12 \mathrm{~W}$ of laser radiation at $935 \mathrm{~nm}$ with an optical-to-optical efficiency of 5.7\% [18]. Recently Li et al. reported $74 \mathrm{~mW}$ of laser radiation from a passively Qswitched Nd:CNGG laser with a repetition rate of $3.788 \mathrm{kHz}$ with a poor optical-to-optical efficiency of only $0.7 \%$ [19].

However, these materials have not yet been investigated to a stage that allows their use in practical lidar systems. The purpose of this paper, therefore, is the development of a highly efficient, diode-pumped and single-frequency laser that uses Nd:YGG $\left(\mathrm{Y}_{3} \mathrm{Ga}_{5} \mathrm{O}_{12}\right)$ as the active material to directly generate laser radiation at $935 \mathrm{~nm}$ appropriate for $\mathrm{H}_{2} \mathrm{O}$-DIAL measurements. Furthermore, the intention was to demonstrate that the INNOSLAB-based MOPA-design [20] is a suitable concept for energy upscaling at $100 \mathrm{~Hz}$ repetition rate which can be applied to this kind of laser source based on Nd:YGG. Its performance was studied in detail to investigate the potential and to analyze whether the shortcomings of sequential converter steps can be outperformed with such a system. After our report on first results with a Qswitched single-frequency laser using Nd:YGG as the active medium [21] we now give a detailed description. To the best of our knowledge, these are the first reports of such laser sources.

The paper is structured as follows. At the beginning the requirements for a water-vapor DIAL transmitter are briefly reviewed. Then, a description of the relevant properties of $\mathrm{Nd}$ :YGG is given which are either known from the literature or which had to be measured within the scope of this study. Subsequently, the setup of the oscillator and its detailed characterization are described, followed by the description of the amplifier setup and characterization of the beam properties of the entire MOPA system.

\section{Requirements}

The requirements of the corresponding laser transmitter for spaceborne $\mathrm{H}_{2} \mathrm{O}$ DIAL were studied within the scope of the assessment report of the WALES (WAter vapor Lidar Experiment in Space) mission [9]. An overview of the requirements is given in Table 1. Generally, in a DIAL system laser pulses of at least two different wavelengths (on-line and offline) are needed. The on-line pulse is spectrally matched to a high absorption line of the molecule to be detected while the off-line pulse is matched to a low absorption line for reference $\left(\lambda_{4}\right)$. For the spaceborne water-vapor DIAL system three on-line wavelengths $\left(\lambda_{1}, \lambda_{2}, \lambda_{3}\right)$ that correspond to different absorption cross sections were proposed, each one especially adapted to a restricted altitude range of the atmosphere to cover the whole troposphere. Consequently, all of these wavelengths will have to be addressed by the DIAL transmitter sequentially. Furthermore, single-frequency operation with a spectral purity of better than $99.9 \%$ is needed 
Table 1 Requirements of the laser source for WALES [9]

\begin{tabular}{lll}
\hline Parameter group & Parameter & Value \\
\hline Spectral & Emission wavelength & $\lambda_{1}=935.685 \mathrm{~nm}$ \\
& & $\lambda_{2}=935.561 \mathrm{~nm}$ \\
& $\lambda_{3}=935.906 \mathrm{~nm}$ \\
& & $\lambda_{4}=935.852 \mathrm{~nm}$ \\
& & $<60 \mathrm{MHz}$ \\
& Frequency stability & $<160 \mathrm{MHz}$ \\
& Laser linewidth & $99.9 \%$ \\
Energetic & Laser spectral purity & \\
Temporal & Pulse energy & $\geq 72 \mathrm{~mJ}$ \\
& $\begin{array}{l}\text { Repetition frequency per } \\
\text { wavelength }\end{array}$ & $\geq 25 \mathrm{~Hz}$ \\
& $\begin{array}{l}\text { Raw data altitude resolution } \\
\text { (limits the maximum pulse }\end{array}$ & $50 \mathrm{~m}$ \\
& $\begin{array}{l}\text { duration) } \\
\text { Beam propagation factor } \mathrm{M}^{2}\end{array}$ & $<3$ \\
\hline
\end{tabular}

to reduce the systematic error of the water-vapor measurement. To enable the required raw data altitude resolution the pulse duration should be in the order of $\sim 200 \mathrm{~ns}$ or less [5].

\section{Properties of Nd:YGG}

\subsection{Classification and Introduction}

Like the ubiquitous laser host material $\mathrm{YAG}\left(\mathrm{Y}_{3} \mathrm{Al}_{5} \mathrm{O}_{12}\right)$, YGG (Yttrium Gallium Garnet, $\mathrm{Y}_{3} \mathrm{Ga}_{5} \mathrm{O}_{12}$ ) also belongs to the group of garnet crystals that follow the stoichiometric relation $\mathrm{C}_{3} \mathrm{~A}_{2} \mathrm{D}_{3} \mathrm{O}_{12}$, where $\mathrm{C}$ sits on the dodecahedral site, $\mathrm{A}$ on the octahedral site and D on the tetrahedral site. Garnets are known to offer beneficial optical and mechanical properties for laser crystals. They are comparatively hard, optically isotropic and have a good thermal conductivity that allows them to be used in high power lasers. Neodymium, a lanthanide, is normally trivalent in a crystal matrix. The 4fsubshell of the trivalent Nd ion, which is relevant for most of the laser transitions, is efficiently shielded from the crystal field by the full subshells $5 \mathrm{~s}$ and $5 \mathrm{p}$. Therefore, narrow emission linewidths can be achieved. The energy of the electronic states of the $\mathrm{Nd}$ ions is defined via the Stark effect by the electric field at the site of the $\mathrm{Nd}$ ion. Modifying the host crystal results in an energy shift of the electronic states and, thus, also in a shift of the emission wavelengths. The peak emission of the $R_{2}-Z_{5}$ transition is at $935.3 \mathrm{~nm}$ (vac.) in $\mathrm{Nd}$ :YGG and covers some absorption lines of water vapor that can be relevant for DIAL systems.

A certain set of crystal parameters have to be known for a proper laser design. Since, when compared to established laser materials, $\mathrm{Nd}$ : YGG is poorly investigated, relevant material parameters needed for the design of the laser had to be determined. This was an essential part of this study.
The proper design of the energy flux inside the laser crystal and the design of spectral filters inside the cavity are based on emission cross-section data for the desired laser wavelength at $935 \mathrm{~nm}$ and of strong neighboring transitions.

Walsh et al. [10] carried out accurate measurements of the emission cross sections with spectroscopic methods. Furthermore, the absorption cross sections for the pump light are needed for the selection of the crystal length and the Nd doping. The lifetime of the upper multiplet limits the storage efficiency and influences the reasonable pumping time. This parameter was also measured by Walsh et al. [10]. The refractive power of the thermal lens is a further crucial parameter for the laser design. Calculations of the refractive power at given thermal loads require at least the $d n / d T$ value as well the thermal conductivity $K_{c}$. While values for $K_{c}$ can be found in literature [22, 23], to the best of our knowledge no values for $d n / d T$ especially at $935 \mathrm{~nm}$ have been published to date. Therefore, this parameter was determined. In this section the relevant laser parameters will be presented in detail and are summarized at the end.

\subsection{Cross sections}

In general, the spectral properties of Nd:YGG are comparable to those of Nd:YAG. The energy level diagram of Nd:YGG is given in Fig. 1. The appropriate effective emission cross sections are depicted for the relevant transitions from the ${ }^{4} \mathrm{~F}_{3 / 2}$ to the ${ }^{4} \mathrm{I}_{9 / 2}$ and the ${ }^{4} \mathrm{I}_{11 / 2}$ multiplet for the emission around $935 \mathrm{~nm}$ and $1062 \mathrm{~nm}$, respectively, in Fig. 2. The data for the emission cross sections were taken from Walsh et al. [10]. Like in Nd:YAG, the $\mathrm{R}_{2}-\mathrm{Y}_{3}$ is the strongest transition in Nd:YGG. In Nd:YGG the emission wavelength is shifted to $1062 \mathrm{~nm}$, compared to the corresponding transition in Nd:YAG at $1064 \mathrm{~nm} .935 \mathrm{~nm}$ and $938 \mathrm{~nm}$ are generated by the $R_{2}-Z_{5}$ and $R_{1}-Z_{5}$ transition, respectively. In contrast to the $\mathrm{Y}_{3}$ level, $\mathrm{Z}_{5}$ has a significant thermal population of about $0.9 \%$ at room temperature. That is the reason why this constellation is also called a quasithree-level system.

Like Nd:YAG, Nd:YGG can be pumped with common laser diodes at $806 \mathrm{~nm}$. The absorption spectrum and absorption cross section around $806 \mathrm{~nm}$ was measured at the Institute of Laser-Physics of the University of Hamburg [24] (see Fig. 3). The values are scaled to an $\mathrm{Nd}$ doping level of $1 \mathrm{at} \%$. As the spectral width of typical diode lasers around $806 \mathrm{~nm}$ is in the range of a few nanometers; the effective absorption is reduced compared to the peak values. For the spectral energy distribution of the laser diode a normalized Gaussian shape

$$
\begin{aligned}
& E d(\lambda)=\frac{2 \sqrt{\ln (2)}}{\sqrt{\pi} \Delta \lambda_{f w h m}} \exp \left(-\frac{\left(\lambda-\lambda_{0}\right)^{2}}{\Delta \lambda_{f w h m}^{2}} 4 \ln (2)\right) \\
& \int_{0}^{\infty} E d(\lambda) d \lambda=1
\end{aligned}
$$




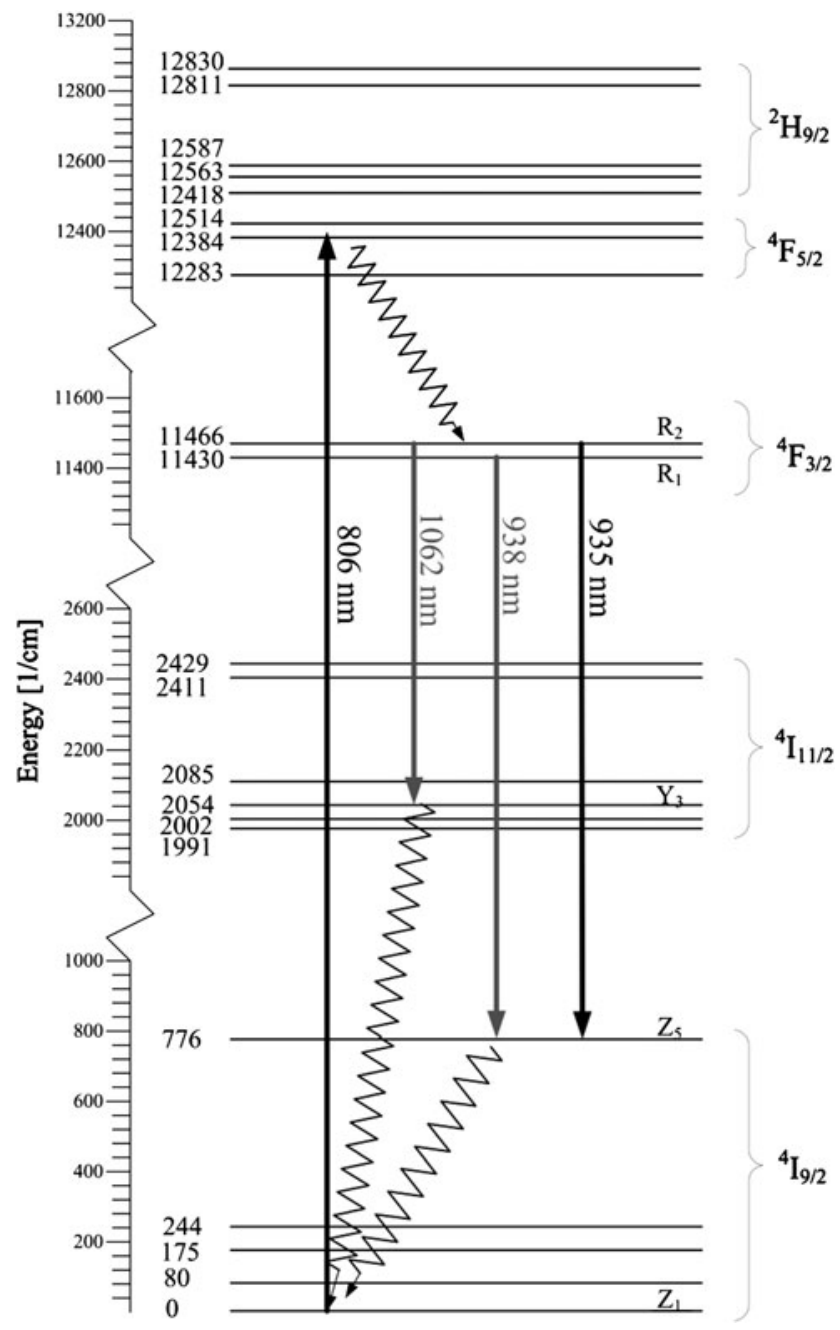

Fig. 1 Energy levels of Nd:YGG. The data for the manifolds ${ }^{4} \mathrm{I}_{9 / 2}$, ${ }^{4} \mathrm{I}_{11 / 2},{ }^{4} \mathrm{~F}_{3 / 2}$ at $295 \mathrm{~K}$ were taken from [10], the data for ${ }^{4} \mathrm{~F}_{5 / 2},{ }^{2} \mathrm{H}_{9 / 2}$ at $77 \mathrm{~K}$ were taken from [26]. The manifolds ${ }^{4} \mathrm{I}_{13 / 2}$ and ${ }^{4} \mathrm{I}_{15 / 2}$ lie within the breaks of the ordinate and are not shown here

was assumed, where $\Delta \lambda_{f w h m}$ and $\lambda_{0}$ are the spectral width and the center wavelength, respectively. The effective absorption coefficient was calculated by

$\tilde{\alpha}\left(\lambda_{0}\right)=-\ln \left(\int_{0}^{\infty} E d(\lambda) \exp \left(-\alpha(\lambda) l_{C}\right) d \lambda\right) / l_{C}$

for a crystal length of $l_{C}=10 \mathrm{~mm}$ and different spectral widths $\Delta \lambda_{f w h m}$ (in the range of $1-3 \mathrm{~nm}$ ). The maximum effective absorption is shifted from $808 \mathrm{~nm}$ to about $806 \mathrm{~nm}$. The maximum effective absorption coefficient for a Gaussian profile with a spectral width of $3 \mathrm{~nm}$, which is a typical bandwidth for pulsed diode laser bars, is $0.3 \mathrm{~mm}^{-1}$.

\subsection{Measurement of $d n / d T$}

The main contribution to the thermal lens in pumped laser crystals is usually given by the change of the refraction index with temperature $d n / d T$. While this parameter is well

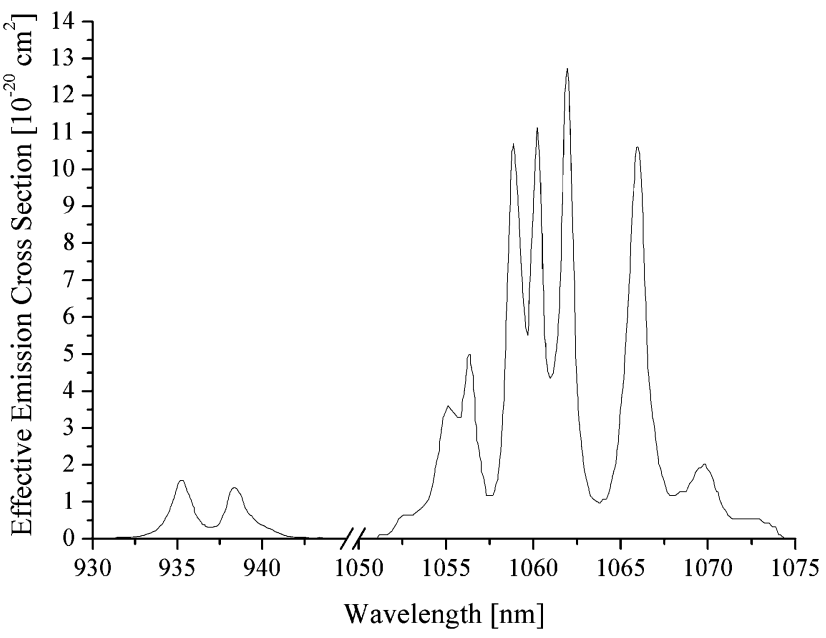

Fig. 2 Effective emission cross section of Nd:YGG from the ${ }^{4} \mathrm{~F}_{3 / 2}$-manifold at room temperature [10]

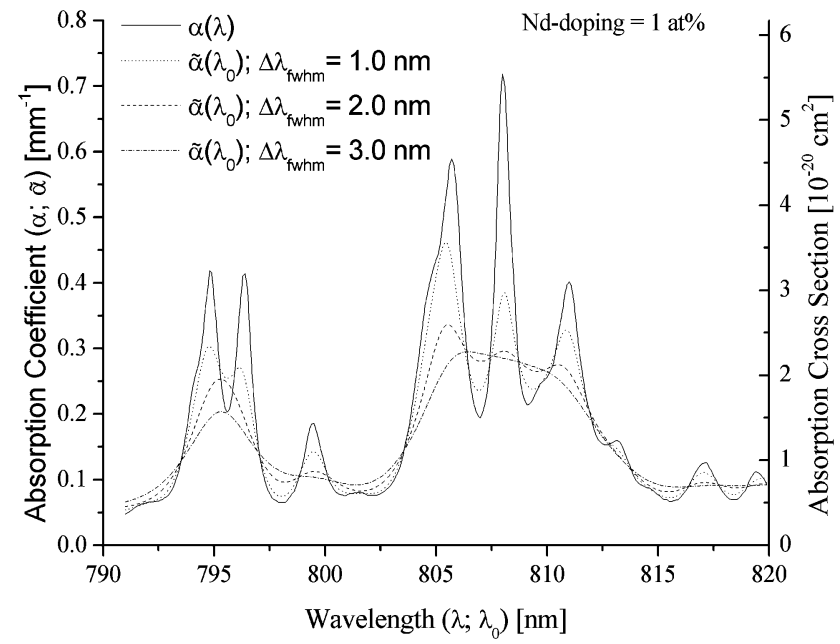

Fig. 3 Measurement of the absorption coefficient and absorption cross section of Nd:YGG; Values are scaled to a Nd doping level of 1 at $\%$ [24]

known for common laser host materials like YAG, no values for YGG especially at $935 \mathrm{~nm}$ were found in the literature.

The $d n / d T$-value was measured using the following setup (see Fig. 4). A prism of $1 \mathrm{~mm}$ height was made from the Nd:YGG boule with an apex angle of about $30^{\circ}$ between the two polished surfaces. The prism was mounted between two copper heat sinks which were contacted to the top and bottom base faces with indium to ensure homogeneous temperature profiles inside the crystal.

The copper heat sink was heated to different temperatures $T=T_{0}+\Delta T$ between $T_{0}=22^{\circ} \mathrm{C}$ and $T_{0}+\Delta T=130^{\circ} \mathrm{C}$ controlled by Pt100-temperature sensors. Radiation from an external cavity diode laser (tuned to $935 \mathrm{~nm}$ ) was applied perpendicular to the entrance surface of the prism. The beam was refracted by the exit surface at an angle $\gamma(T)$ relative to the surface normal. $\gamma(T)$ can be expressed by a sum of two 


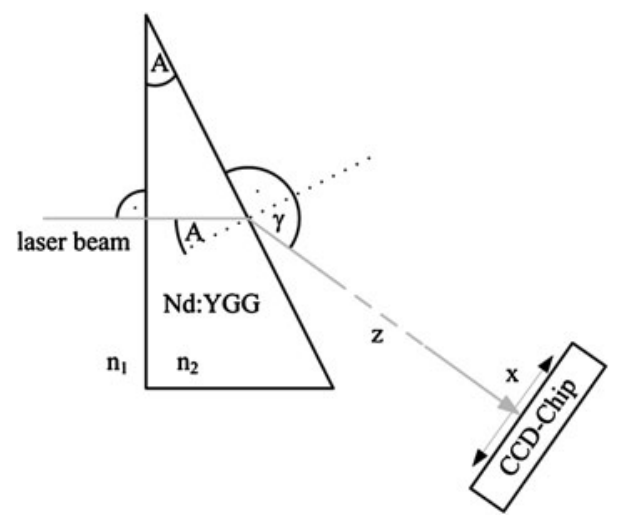

Fig. 4 Experimental setup for $d n / d T$ measurements. The variation of the refraction angle is measured with a CCD camera

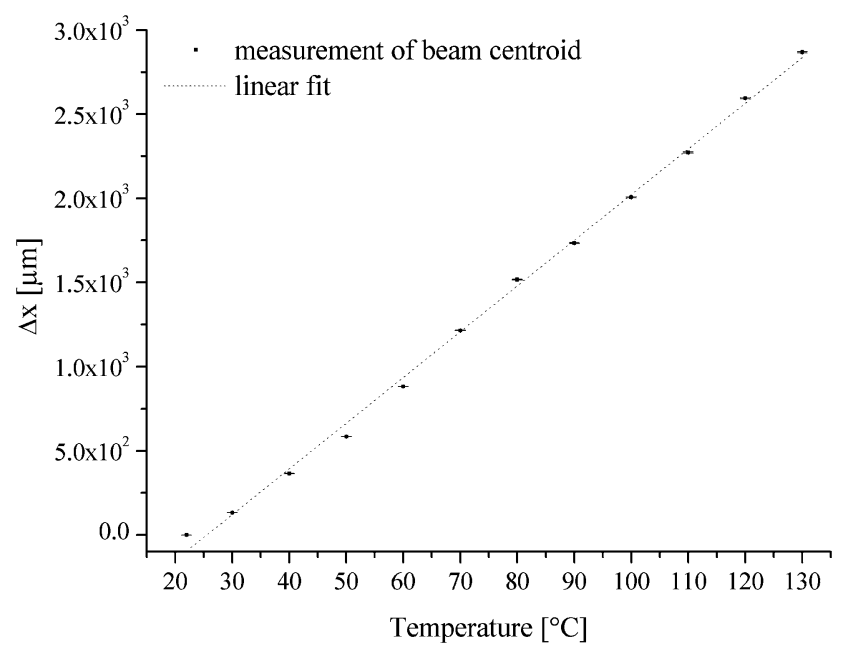

Fig. 5 Measurement of the beam centroid on the CCD-chip versus temperature and linear data fit

angles by $\gamma\left(T_{0}+\Delta T\right)=\gamma\left(T_{0}\right)+\Delta \gamma(\Delta T)$, where the first one depends only on the temperature $T_{0}$ and the second one only on the temperature change $\Delta T$. The change in refraction angle with temperature $\Delta \gamma(\Delta T)$ was monitored as a shift of the beam position (centroid of the intensity distribution) on the chip of a CCD camera $\Delta x=\Delta \gamma / z$ at a distance of $z=750 \mathrm{~mm}$ to the prism. For each temperature value, a set of position measurements was averaged to compensate the position jitter caused by temperature fluctuations in the surrounding air. The $\Delta x(T)$-data were fitted linearly to obtain the gradient $d \Delta x / d T$ (see Fig. 5).

A formula was derived from Snell's law, which allows the calculation of $d n / d T$ from the gradient $d \Delta x / d T$. Therefore, the temperature dependent refraction index was substituted by $n\left(T_{0}+\Delta T\right)=n\left(T_{0}\right)+\Delta n(\Delta T)$, where $n\left(T_{0}\right)$ is the refractive index at temperature $T_{0}$, and $\Delta n(\Delta T)$ is a small difference in refraction index caused by the temperature deviation $\Delta T$. Making the derivative of Snell's law with respect to temperature, using the following approxima-
Table 2 Values that were used to calculate $d n / d T$

\begin{tabular}{ll}
\hline Parameter & Value \\
\hline$A$ & $30^{\circ} 25^{\prime} \pm 5^{\prime}$ \\
$z$ & $(750 \pm 5) \mathrm{mm}$ \\
$n_{1}$ & $1.0003 \pm 0.0002[25]$, estimated error \\
$n_{2}\left(T_{0}\right)$ & $1.915 \pm 0.005[26]$, estimated error \\
$d \Delta x / d T$ & $(27.156 \pm 0.410) \mu \mathrm{m} /{ }^{\circ} \mathrm{C}$ \\
\hline
\end{tabular}

tion for small angles $(\Delta \gamma \ll 1) \sin (\gamma)=\sin \left(\gamma_{0}+\Delta \gamma\right)=$ $\sin \left(\gamma_{0}\right) \cos (\Delta \gamma)+\cos \left(\gamma_{0}\right) \sin (\Delta \gamma) \approx \sin \left(\gamma_{0}\right)+\cos \left(\gamma_{0}\right) \Delta \gamma$ and substituting $\gamma_{0}\left(T_{0}\right)=\arcsin \left[n\left(T_{0}\right) / n_{1} \sin (A)\right]$ according to Snell's law yields

$$
\begin{aligned}
\frac{d n}{d T} & =\frac{d}{d T}\left(\frac{\sin (\gamma(T))}{\sin (A)} n_{1}\right)=\frac{\cos \left(\gamma_{0}\left(T_{0}\right)\right) n_{1}}{\sin (A)} \frac{d}{d T}(\Delta \gamma(\Delta T)) \\
& =\frac{\cos \left(\arcsin \left[n\left(T_{0}\right) / n_{1} \sin (A)\right]\right) n_{1}}{\sin (A) z} \frac{d \Delta x}{d T}
\end{aligned}
$$

Here $n_{1}$ is the refractive index of the surrounding air.

$d n / d T$ was calculated using the values listed in Table 2 to

$$
\begin{aligned}
& d n / d T_{\mathrm{Nd}: \mathrm{YGG}}=(17.6 \pm 0.8) \times 10^{-6} \mathrm{~K}^{-1} \\
& \left(\text { for } 22^{\circ} \mathrm{C}<T<130^{\circ} \mathrm{C}\right) .
\end{aligned}
$$

\subsection{Crystal properties and design issues}

In this section a summary of the relevant crystal properties of Nd:YGG is given, which is based on the measurements presented before and on literature citations. From this, certain consequences for the laser design are deduced. For better understanding, the crystal parameters of Nd:YGG (see Table 3) are also compared to those of Nd:YAG (see Table 4). In contrast to Nd:YGG, Nd:YAG is widely used and well investigated. Furthermore, alternative laser sources at $935 \mathrm{~nm}$ such as OPOs and Ti:Sapphire converters are usually pumped at $532 \mathrm{~nm}$ with frequency doubled Nd:YAG lasers. Consequently, the performance of these competing laser concepts strongly depend on the performance of the $\mathrm{Nd}$ :YAG pump laser.

It can easily be deduced from the fundamental crystal parameters, especially the appropriate emission cross sections and thermo-optical properties, that Nd:YGG-based lasers at $935 \mathrm{~nm}$ cannot compete with comparable Nd:YAG-based sources at $1064 \mathrm{~nm}$ regarding their performance. But it is not directly evident whether these sources can compete with OPO or Ti:Sapphire systems emitting at $935 \mathrm{~nm}$. In the two latter cases the overall efficiency is reduced significantly as two further converter steps are needed to generate radiation at $935 \mathrm{~nm}$. At the end of this article, state-of-the-art overall conversion efficiencies for these systems will be discussed. 
Table 3 Summary of relevant crystal properties of $\mathrm{Nd}: \mathrm{YGG}$
Table 4 Some relevant crystal properties of Nd:YAG

\begin{tabular}{|c|c|c|c|}
\hline Parameter & Description & Value & Reference \\
\hline$f_{b}$ & Thermal population of $Z_{5}$ & $0.9 \%$ at $293 \mathrm{~K}$ & $\begin{array}{l}\text { Calculated from energy } \\
\text { levels given by [10] }\end{array}$ \\
\hline$f_{a}$ & Thermal population of $R_{2}$ & $46 \%$ at $293 \mathrm{~K}$ & $\begin{array}{l}\text { Calculated from energy } \\
\text { levels given by [10] }\end{array}$ \\
\hline$\sigma_{935}$ & $\begin{array}{l}\text { Effective emission cross section } \\
\text { of } \mathrm{R}_{2}-\mathrm{Z}_{5} \text { transition: }\end{array}$ & $1.5 \times 10^{-20} \mathrm{~cm}^{2}$ & {$[10]$} \\
\hline$\sigma_{938}$ & $\begin{array}{l}\text { Effective emission cross section } \\
\text { of } \mathrm{R}_{1}-\mathrm{Z}_{5} \text { transition: }\end{array}$ & $1.43 \times 10^{-20} \mathrm{~cm}^{2}$ & {$[10]$} \\
\hline$\sigma_{1062}$ & $\begin{array}{l}\text { Effective emission cross section } \\
\text { of strongest transition } \mathrm{R}_{2}-\mathrm{Y}_{3} \text { : }\end{array}$ & $11.4 \times 10^{-20} \mathrm{~cm}^{2}$ & {$[10]$} \\
\hline$F$ sat 935 & Saturation fluence at $935 \mathrm{~nm}$ & $14 \mathrm{~J} / \mathrm{cm}^{2}$ & Calculated from $\sigma_{935}$ \\
\hline$F$ sat938 & Saturation fluence at $938 \mathrm{~nm}$ & $15 \mathrm{~J} / \mathrm{cm}^{2}$ & Calculated from at $\sigma_{938}$ \\
\hline$F$ sat $_{1062}$ & Saturation fluence at $1062 \mathrm{~nm}$ & $1.6 \mathrm{~J} / \mathrm{cm}^{2}$ & Calculated from $\sigma_{1062}$ \\
\hline$\alpha_{808}$ & $\begin{array}{l}\text { Absorption at } 806 \mathrm{~nm} \text {, width } \\
\text { fwhm: } 2-3 \mathrm{~nm}, \mathrm{Nd} \text { doping: } \\
1 \text { at } \%\end{array}$ & $0.3 \mathrm{~mm}^{-1}$ & $\begin{array}{l}\text { Calculated from measured } \\
\text { absorption spectrum }\end{array}$ \\
\hline$\alpha_{935}$ & $\begin{array}{l}\text { Absorption at } 935 \mathrm{~nm} \text {, } \\
T=293 \mathrm{~K}, \text { Nd doping: } 1 \text { at } \%\end{array}$ & $3.79 \mathrm{~m}^{-1}$ & Calculated from $\sigma_{935}$ \\
\hline$d n / d T$ & & $(17.6 \pm 0.8) \times 10^{-6} \mathrm{~K}^{-1}$ & Measured in this work \\
\hline$K_{c}$ & Thermal conductivity & $9 \mathrm{~W} / \mathrm{m} / \mathrm{K}$ & {$[23]$} \\
\hline$\tau_{a}$ & $\begin{array}{l}\text { Lifetime of upper laser level } \\
{ }^{4} \mathrm{~F}_{3 / 2}\end{array}$ & $254 \mu \mathrm{s}$ & {$[27]$} \\
\hline
\end{tabular}

Nd:YGG offers a comparatively small emission cross section at the desired wavelength at $935 \mathrm{~nm}$ of about $1.5 \times$ $10^{-20} \mathrm{~cm}^{2}$. This leads to a saturation fluence of about $14 \mathrm{~J} / \mathrm{cm}^{2}$. In comparison, the saturation fluence in Nd:YAG at the peak emission of $1064 \mathrm{~nm}$ is only about $0.7 \mathrm{~J} / \mathrm{cm}^{2}$. Consequently, in Nd:YGG at $935 \mathrm{~nm}$, fluences that are 20 times higher are needed than in Nd:YAG at $1064 \mathrm{~nm}$ to achieve equal extraction efficiencies. As the damage thresh- old of optical surfaces usually is in the range of $10 \mathrm{~J} / \mathrm{cm}^{2}$ efficient energy extraction is limited by damage threshold in Nd:YGG, whereas this is not an issue for Nd:YAG at $1064 \mathrm{~nm}$.

Like in Nd:YAG the $\mathrm{R}_{2}-\mathrm{Y}_{3}$ transition at $1062 \mathrm{~nm}$ in Nd:YGG offers the highest emission cross section of $11.4 \times$ $10^{-20} \mathrm{~cm}^{2}$, which is about 7.6 times higher than at $935 \mathrm{~nm}$. As both of these transitions have the same upper laser level, 
special care has to be taken to avoid energy losses by amplified spontaneous emission or parasitic lasing at $1062 \mathrm{~nm}$. Additionally, the ground level of the $\mathrm{R}_{2}-\mathrm{Z}_{5}$ transition is thermally occupied with $0.9 \%$ at room temperature. That is the reason why this transition is called a quasi-three-level system. Thermal population of the ground level demands a certain amount of pump energy to achieve transparency at the laser wavelength before gain can be created in the crystal. Because of this thermal population of the lower laser level, the upper laser level needs to be more strongly populated compared to four-level-lasers, which, on the other hand, increases the gain for $1062 \mathrm{~nm}$ emission as well.

At $300 \mathrm{~K}$ the undoped YGG crystal possesses a thermal conductivity of $9 \mathrm{~W} / \mathrm{m} / \mathrm{K}$ [23], being 1.5 times lower than in $\mathrm{Nd}$ :YAG $(14 \mathrm{~W} / \mathrm{m} / \mathrm{K})$ [28]. Furthermore, the change of the refraction index with temperature $d n / d T$ is about 2.4 time stronger in Nd:YGG than in Nd:YAG. Consequently, this means that in equally pumped laser crystals thermal lensing is about a factor of 3.7 stronger in Nd:YGG than in Nd:YAG. Furthermore, there are two requirements leading to small pump spots inside the laser crystal which might cause even shorter thermal lenses. On the one hand, high-energy fluences are needed for efficient energy extraction. This leads to small laser modes and, consequently, to small pump spots. On the other hand, high pump intensities are required to compensate for the population of the lower laser level.

In spite of several undesirable properties of $\mathrm{Nd}: \mathrm{YGG}$ at $935 \mathrm{~nm}$ in comparison to, for example, Nd:YAG at $1064 \mathrm{~nm}$, laser emission with remarkable efficiency is possible. Several sources that have been realized at such low gain transitions demonstrate their potential. Moreover, high saturation fluences are not an inherent problem concerning extraction efficiency. High quality coatings optimized for high damage thresholds might withstand these problems. But nevertheless, it is a challenging issue to find a well-balanced parameter setup for efficient and stable Q-switched lasers at $935 \mathrm{~nm}$ in Nd:YGG.

\section{System design}

Based on the experimental findings described above, the Nd:YGG laser was designed as a MOPA arrangement in order to isolate the generation of the spectral, temporal and spatial from the energetic beam properties.

This approach allows for generation of high pulse energies in the nanosecond-regime with nearly diffractionlimited beam qualities. Low energy pulses were generated in a stabilized injection seeded Q-switch oscillator. The energy was scaled in a single amplifier stage following the well established INNOSLAB design, which is well known to achieve a high pulse energy of about $100 \mathrm{~mJ}$ and repetition rate of $100 \mathrm{~Hz}$ at very high overall efficiency [29].

\subsection{Oscillator}

\subsubsection{Setup}

The oscillator setup is depicted in Fig. 6. The crystal was an Nd:YGG rod with a diameter of $3 \mathrm{~mm}$ and a length of $10 \mathrm{~mm}$. The Nd-doping level was 1 at\% (percentage of the yttrium ions substituted by $\mathrm{Nd}$ ions). It was pumped from two sides by fiber-coupled passively cooled diode bars. The end of the fiber with a core-diameter of $400 \mu \mathrm{m}$ and a numerical aperture of 0.22 was imaged into the crystal by means of a 1:2 telescope. Therefore, the pump spot radius $\omega_{p}$ inside the crystal was about $400-500 \mu \mathrm{m}$. Both optical faces of the crystal had an antireflex coating for the laser wavelength at $935 \mathrm{~nm}$, for the pump wavelength at $806 \mathrm{~nm}$ and at $1062 \mathrm{~nm}$ to avoid parasitic lasing at the strongest transition of the $\mathrm{Nd}^{3+}$ ion. In all experiments the duration of the pump pulses was $200 \mu$ s.

A plano-convex mirror configuration was used for the cavity. It was optimized to achieve dynamical stability as described in Magni [30] at a certain working point. The plane outcoupler ensures that the beam waist position is fixed at the plane outcoupler surface. Its reflectivity for the laser beam at $935 \mathrm{~nm}$ is $80 \%$. The concave mirror compensates for the thermal lens inside the laser rod which has a focal length of down to $200 \mathrm{~mm}$. The smallest spot size of the beam is on the convex end mirror, which has a high reflective coating with high damage threshold. Both, end mirror and outcoupler as well as the pump mirrors had a low reflectivity at the strongest transition at $1062 \mathrm{~nm}$ to suppress lasing at this wavelength.

A thin film polarizer (TFP) and a BBO-Pockels cell were sited in the low fluence arm of the resonator to prevent their optical damage. The eigenpolarization of the cavity is in sdirection with respect to the TFP and pumping mirrors. The TFP offers higher reflection for $s$-polarization than transmission for $p$-polarization. Furthermore, the pumping mirrors have lower losses for $s$-polarization, as well.

Two quarter-wave plates were added between the pumping mirrors and laser crystal in order to achieve twistedmode operation. This is necessary in linear cavities to avoid spatial hole burning caused by standing waves [31]. The quarter-wave plates have an antireflex coating at both laser and pump wavelength at $935 \mathrm{~nm}$ and $806 \mathrm{~nm}$, respectively. Siting the quarter-wave plates outside the pump path would certainly lead to higher pumping efficiencies, but would have reduced the overall reflectivity of the pumping mirrors for the resonator mode as their reflectivity for $p$-polarization is reduced.

In order to achieve single-longitudinal-mode operation, the oscillator was seeded with a fiber-coupled DFB-diode laser. The light was coupled into the cavity in $p$-polarization through the TFP and was geometrically matched to the 
Fig. 6 Schematic oscillator setup

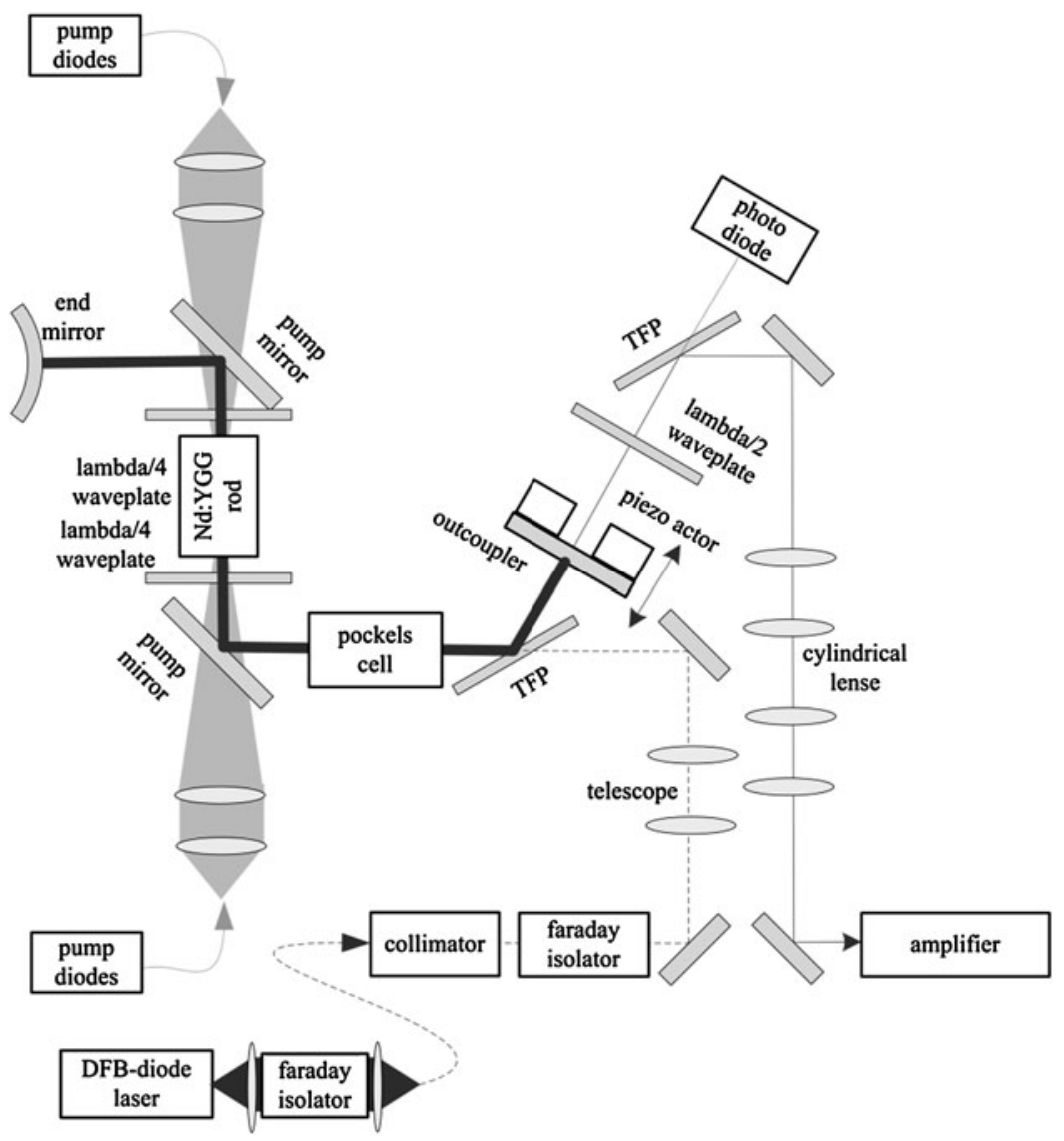

eigenmode of the cavity via a simple spherical telescope. The major part of the seed laser beam is converted into $s$-polarization by two Pockels-cell passes if the quarter-wave voltage is applied during the pumping period. Thus, a certain percentage of the light is kept inside the cavity. If the oscillator cavity is in resonance with the seed light, its intensity inside the cavity is maximal due to self-interference. Consequently, the light signal which leaks from the cavity through the outcouple mirror also has a maximum. This light signal was measured with an amplified photodiode. The signal strength was controlled with a beam splitter consisting of a half wave plate and a TFP. This was necessary to protect the photodiode from optical damage as the laser pulses propagated along the same optical path as the seed laser beam.

The seed laser could be shifted to different center wavelengths via temperature tuning. It was protected from depolarized laser pulses from the cavity by two $30 \mathrm{~dB}$ Faraday isolators in order to avoid damage and spectrally unstable operation.

Stabilization of the cavity length was done with the rampand-fire-method $[32,33]$. A low-voltage piezo ring at the outcoupler was used to ramp the cavity. At the end of the pump period a resonance event is detected by the ramp-andfire electronics which generates a TTL signal for the QS driver. More details of the stabilization techniques used can be found in [33].

Finally, the oscillator beam is mode matched to the astigmatic INNOSLAB-amplifier mode using two cylindrical telescopes having perpendicular optical axes.

\subsubsection{Thermal lens measurements}

The knowledge of the refractive power of the thermal lens is a prerequisite for a precise cavity design. But as thermal lens calculations are error prone since the input parameters like quantum efficiency, pump intensity distribution, $d n / d T$-values and thermal conductivity are not known at sufficient accuracy in this case, the focal length of the thermal lens was determined experimentally.

Gradients in temperature profiles induced by crystal pumping usually lead to thermal lensing. The major contribution to the thermal lens is given by the change of the refractive index with temperature $d n / d T$. The focal length of the thermal lens $f_{\text {rod }}$ caused by this effect can be described analytically in laser crystals which are end pumped with a Gaussian profile by

$f_{\text {rod }}=\frac{K_{C}}{d n / d T} \frac{\pi \omega_{P}}{P_{\text {heat }}}$, 


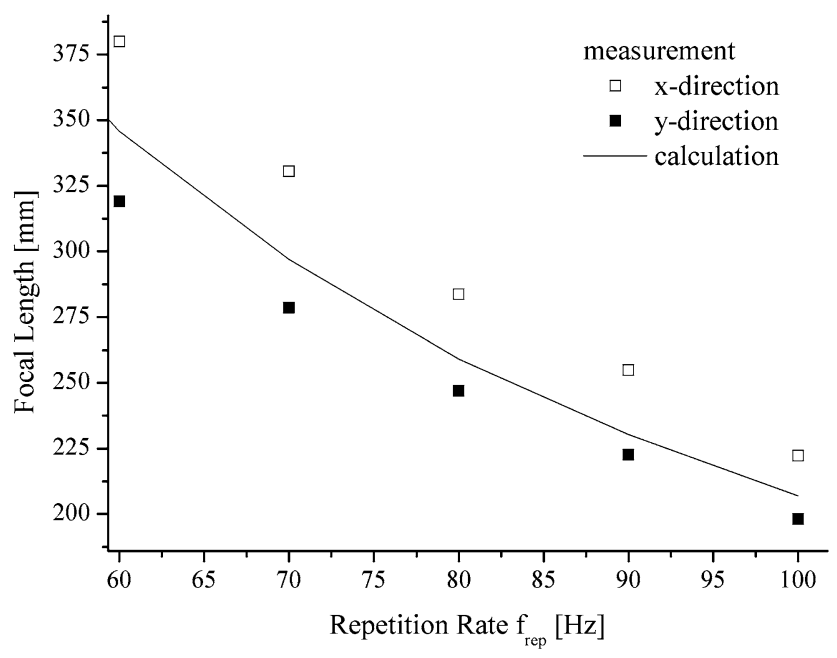

Fig. 7 Thermal lens of an end-pumped Nd:YGG rod; measurements and calculated values

where $K_{C}$ is the thermal conductivity, $\omega_{P}$ the $\left(1 / e^{2}\right)$ Gaussian radius of the pump beam and $P_{\text {heat }}$ the heating power [28]. Here, a radially uniform pump light distribution has been assumed as well as radial heat flow from the rod to a thermal heat sink of fixed temperature. The heating power can be calculated by $P_{\text {heat }}=\mu_{\text {heat }} E_{\text {abs }} f_{\text {rep }}$ from the absorbed pump energy per pulse $E_{\mathrm{abs}}$, the repetition rate of the pump $f_{\text {rep }}$ and the heating efficiency $\mu_{\text {heat }}=\left(1-\mu_{Q} \mu_{\text {stokes }}\right) \cdot \mu_{Q}$ and $\mu_{\text {stokes }}$ are the quantum efficiency and the Stokes efficiency, respectively.

The thermal lens of the end-pumped rod inside the resonator was measured by comparing the measured Rayleigh lengths and waist positions with the theoretical values that were derived by a simple ABCD-matrix formalism in which the rod was represented by a thin lens. This measurement was done for different repetition rates $f_{\text {rep. }}$. For each repetition rate the focal length of the thin lens in the model was adapted to achieve a minimum deviation between the theoretical and measured Rayleigh lengths and waist positions. Different merit functions for the minimization did not significantly change the resulting focal length. The rod was pumped with $6.28 \mathrm{~W}$ mean power at $100 \mathrm{~Hz}$ repetition rate. At this working point the thermal lens has a focal length of about $200 \mathrm{~mm}$. The measured values for the thermal lens could be reproduced well by the theoretical model assuming a quantum efficiency of 0.88 and a pump radius of $400 \mu \mathrm{m}$. Slightly different thermal lenses were observed for the $x$ - and $y$-axis that are parallel and perpendicular, respectively, to the beam plane (see Fig. 7). Different focal lengths might occur when cooling of the crystal is not perfectly symmetric. This effect can be significantly reduced if the laser crystal is soldered into the heat sink as is done for the slab crystal of the amplifier, which will be described later.

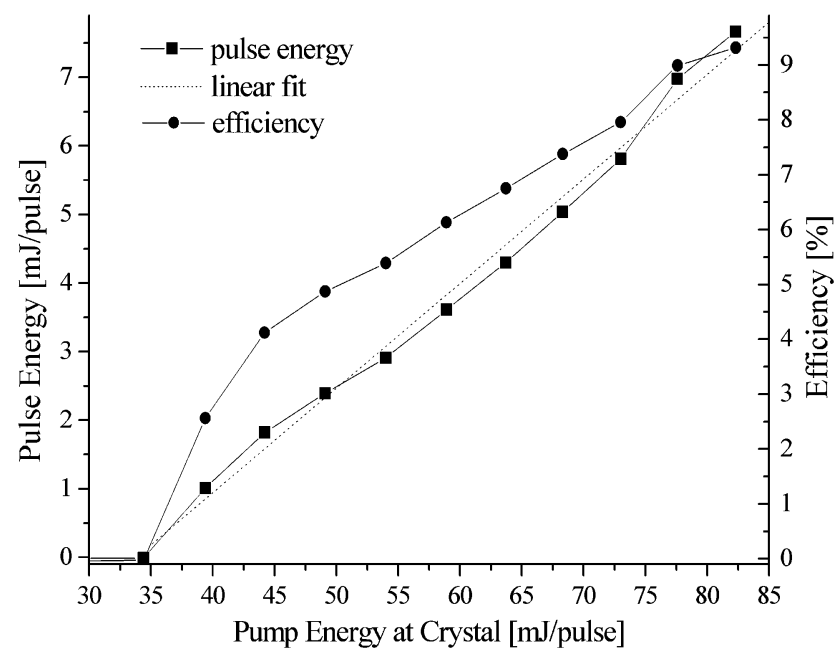

Fig. 8 Pulse energy and efficiency of the oscillator in QS-MM-operation

\subsubsection{Measurement of the beam properties}

QS experiments in longitudinal multimode operation (QS$\mathrm{MM})$ as well as in single-longitudinal-mode operation (QSSLM) were conducted. In QS-MM-operation, $7.7 \mathrm{~mJ}$ were generated with an optical-to-optical efficiency of 9.3\% (pump energy at the crystal-to-laser output). The slope efficiency was measured to be $15.2 \%$. These experiments were done without the two quarter-wave plates for twisted-mode operation (see Fig. 8). For QS-SLM-operation these quarterwave plates were added. Due to the additional losses, the output energy is somewhat lower. At the maximum gain wavelength of $935 \mathrm{~nm}, 4.3 \mathrm{~mJ}$ of pulse energy with an optical-to-optical efficiency of $5.7 \%$ was generated out of $75 \mathrm{~mJ}$ pump energy. The pulse duration at that working point was $52.5 \mathrm{~ns}$.

As stated before, high extraction efficiencies require high-energy fluences inside the laser medium. In order to determine the energy fluence within the resonator, the caustic of the intracavity beam was calculated for different thermal lenses by ABCD-matrix formalism. The mean energy fluence is given by

$F=\frac{E_{p} \kappa}{\pi \omega^{2}}$,

where $E_{p}$ is the pulse energy of the oscillator outside of the cavity, $\omega$ the $1 / e^{2}$-radius of the beam and $\kappa=(1+R) /(1-$ $R$ ) the intracavity enhancement factor for a given outcoupler reflectivity $R$ [28]. It has to be noted that the maximum fluence of the beam is two times higher than $F$. At a thermally induced focal length of $200 \mathrm{~mm}$, which corresponds to the measured value, and at a pulse energy of $4.3 \mathrm{~mJ}$, the energy flux at the crystal, the end mirror and outcoupler is about $13 \mathrm{~J} / \mathrm{cm}^{2}, 21 \mathrm{~J} / \mathrm{cm}^{2}$ and $20 \mathrm{~J} / \mathrm{cm}^{2}$, respectively (see Fig. 9). For larger focal lengths the fluences decrease. The energy 
fluence at the maximum pulse energy of $7.7 \mathrm{~mJ}$ corresponds to a mean energy fluence of as high as $23 \mathrm{~J} / \mathrm{cm}^{2}$ inside the laser crystal.

Due to the high dynamic stability of the resonator, the beam was diffraction limited over a wide energy range (see Fig. 10). Measurements of the beam quality were done in QS-MM-operation. All measurements of the beam radius were performed with the second-moment method according to the international standard test method for laser beam widths, divergence angles and beam propagation ratios, ISO 11146. The beam has a perfect $\mathrm{TEM}_{00}$ shape in near and far field (see Fig. 11).

\subsubsection{Roundtrip losses and simulation}

A simple model based on rate equations was used in order to calculate the energetic and temporal beam properties of

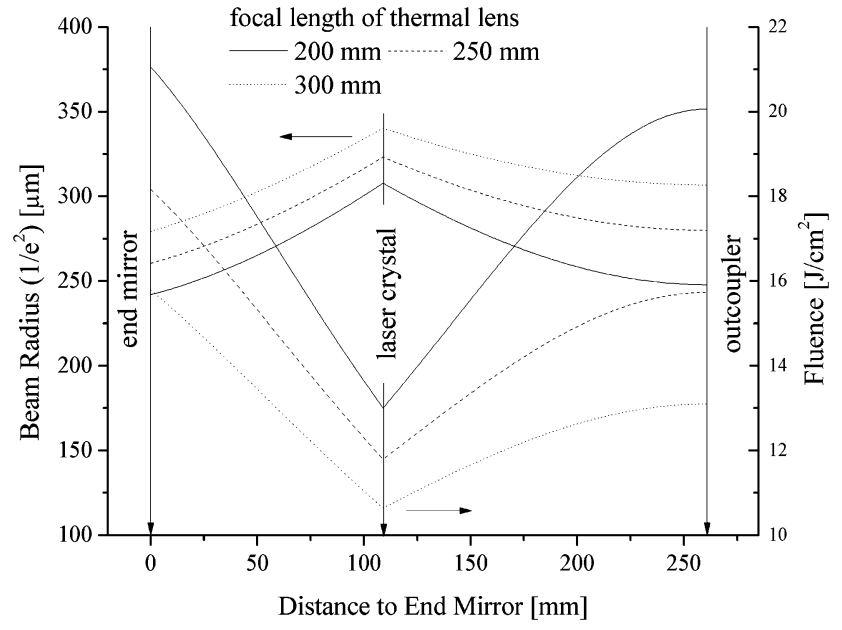

Fig. 9 Calculated beam radii and energy fluences inside the oscillator for different thermal lenses the oscillator out of the pump energy distribution and geometrical cavity properties. This model is an essential tool for the design of highly efficient oscillator setups. But, especially in case of innovative and poorly investigated laser materials, not all properties are sufficiently known for an accurate prediction of pulse energy and duration. This includes, for example, efficiency-decreasing bottleneck factors like the quantum efficiency $\eta_{Q}$ and the inversion reduction factor $\gamma$, which was introduced by Degnan et al. $[34,35]$. Furthermore, the transmission or reflection losses of all intracavity components and the diffraction losses have to be accounted for in order to allow accurate predictions to be made. Fortunately, the total roundtrip loss $L$ of a laser cavity can experimentally be determined by the method described by Findlay and Clay [37]. According to this method, the threshold-pump energy $E_{\text {thres- } i}$ was precisely measured for different reflectivities of the outcoupling mirror $R_{i} \in$

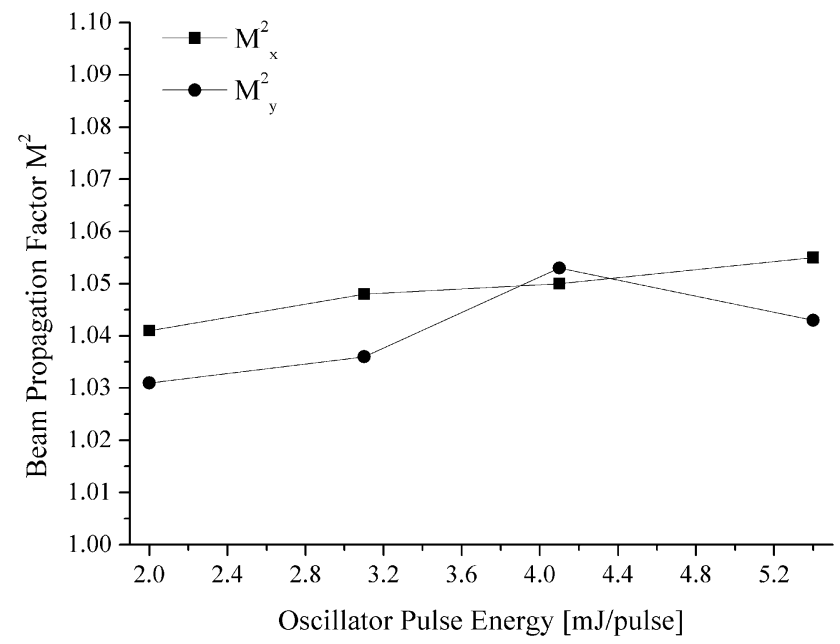

Fig. 10 Beam quality of the oscillator for different pulse energies
Fig. 11 Beam-quality measurement of the oscillator at $5.4 \mathrm{~mJ}$. Beam diameter measurements were done with the second-moment method. At three positions the normalized intensity distribution is also given. All images have equal dimensions

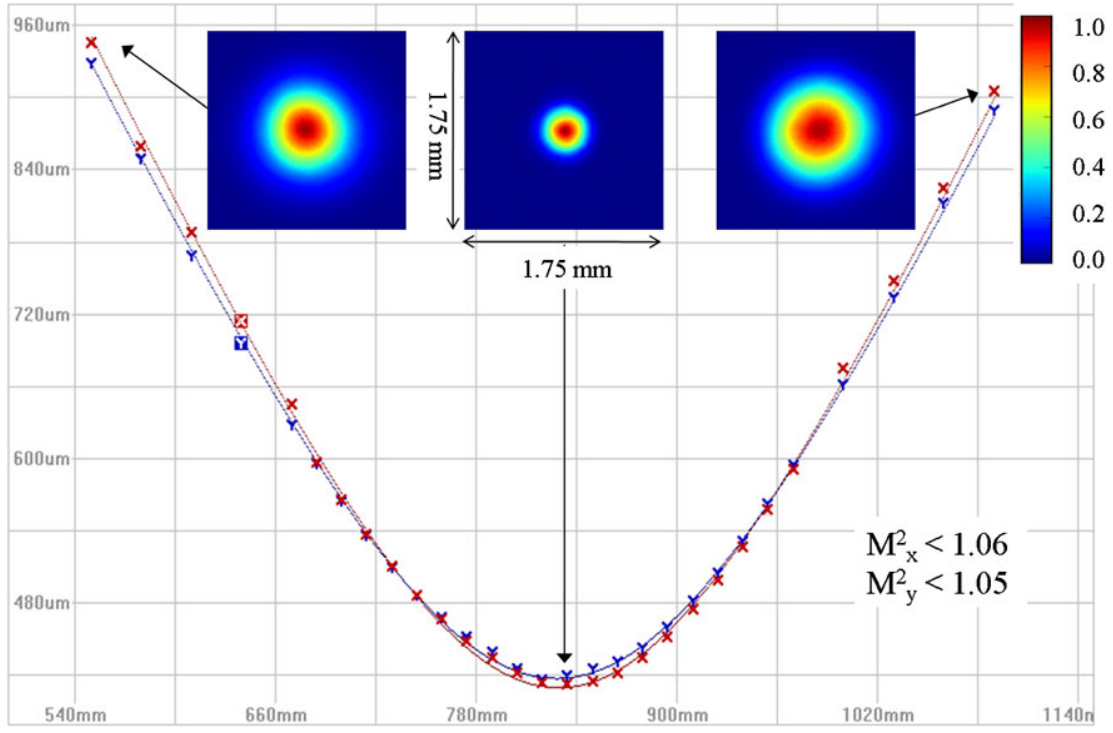




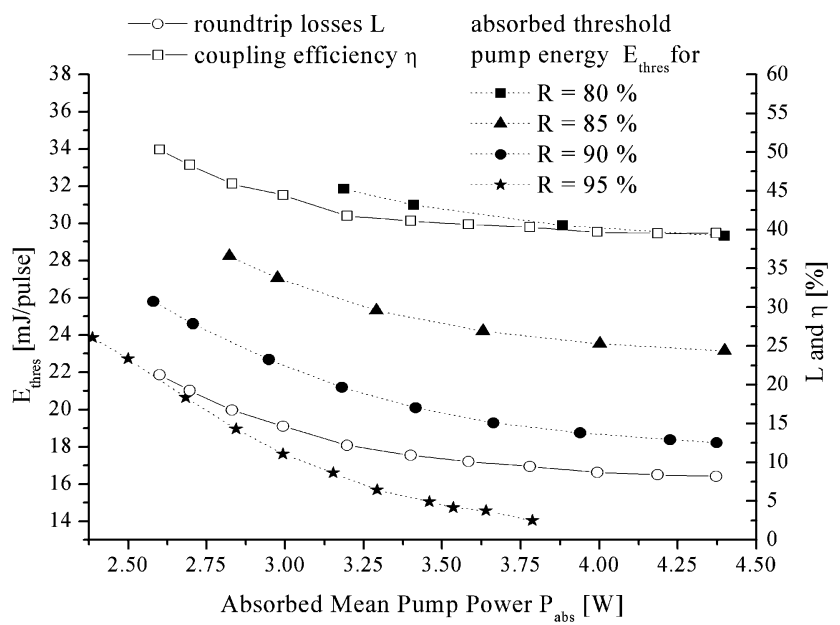

Fig. 12 Measurement of the roundtrip losses $L$ and the total coupling efficiency $\eta$ according to Findlay and Clay [37] for different absorbed mean pump powers and different values of the output coupler's reflectivity

$\{80 \%, 85 \%, 90 \%, 95 \%\}$. By linear fit in the following manner:

$-\ln \left(R_{i}\right)=K E_{\text {thres }-i}-\ln (1-L)$,

the factor $K$ and the roundtrip loss $L$ were determined (see Fig. 12). For a given effective emission cross section $\sigma_{935}$, energy $E_{\mathrm{ph}}$ of the laser photons and pump area $A_{p}$, the total coupling efficiency $\eta=\eta_{\mathrm{st}} \eta_{Q} \eta_{\text {store }} \eta_{M}$ can be calculated from the factor $K=2 \sigma_{935} E_{\mathrm{ph}}^{-1} A_{p}^{-1} \eta$. Here, $\eta_{\mathrm{st}}$ is the Stokes efficiency, $\eta_{Q}$ the quantum efficiency, $\eta_{\text {store }}$ the storage efficiency and $\eta_{M}$ the mode overlap efficiency of laser mode and pump mode, respectively. Unfortunately, the losses $L$ usually depend on the appropriate threshold-pump energy due to the fact that at different mean pump powers different thermal depolarizations and different mode overlaps due to different thermal lenses are induced. Therefore, the measurements were performed at different repetition rates and, thus, different mean pump powers. Finally, the roundtrip losses and the total coupling efficiency $\eta$ were determined at 11 different mean pump powers. Intermediate values for the threshold-pump energies were determined by linear interpolation.

It can be seen that both the total coupling efficiency $\eta$ and $L$ decrease with increasing mean pump power. This is due to the fact that the cavity becomes unstable at low refraction powers of the thermal lens. At this working point the pump spot is small with respect to the cross section of the fundamental laser mode. In this case the mode overlap $\eta_{M}$ but also the refraction losses are comparatively high. Thus, the coupling efficiency $\eta$ and $L$ are high, as well. If the refraction power of the thermal lens increases, both mode overlap and refraction losses decrease. Consequently, also $\eta$ and $L$ decrease. For higher pump powers the losses $L$ ap- proach the reabsorption losses of the unpumped laser crystal at room temperature of $L_{\text {reabs }}=1-\exp \left(-2 l_{c} \alpha_{935}\right) \approx 7.3 \%$.

The pulse energy and temporal pulse shape were calculated using the following rate equations:

$$
\begin{aligned}
& \frac{d n}{d t}(t)=-\gamma^{*} \Gamma(t), \\
& \frac{d \Phi}{d t}(t)=l_{c} A_{p} \Gamma(t)-\Phi(t) \tau_{c}^{-1}, \\
& \Gamma(t)=c \sigma_{\mathrm{eff}} n(t) \Phi(t) l_{R}^{-1} A_{p}^{-1}, \\
& \tau_{c}=-2 l_{R} c^{-1} \ln \left(R\left[1-L\left(P_{\mathrm{abs}}\right)\right]\right)^{-1} .
\end{aligned}
$$

Here, $n$ is the mean inversion density, $\gamma^{*}$ the effective inversion reduction factor, $\Phi$ is the intracavity photon number, $l_{c}$ the length of the laser medium, $c$ the speed of light in vacuum and $R$ the reflectivity of the outcoupler. The inversion density after the pumping process prior the pulse build-up was calculated by $n(t=0)=E_{\mathrm{abs}} \eta\left(P_{\mathrm{abs}}\right) E_{\mathrm{ph}}^{-1} l_{c} A_{p}$ accounting for the power dependent total coupling efficiency. Degnan introduced an expression for a time-dependent inversion reduction factor

$$
\begin{aligned}
\gamma(t)= & e^{-\gamma_{a} t}\left[f_{a}+\left(1-f_{a}\right) e^{-\gamma_{u} t}\right] \\
& +e^{-\gamma_{b} t}\left[f_{b}+\left(1-f_{b}\right) e^{-\gamma_{l} t}\right],
\end{aligned}
$$

where $\gamma_{a}$ and $\gamma_{b}$ are the relaxation rates for the upper and lower multiplet and $\gamma_{u}$ and $\gamma_{l}$ the thermalization rates within the upper and lower multiplet, respectively. $f_{a}$ and $f_{b}$ are the Maxwell-Boltzmann probabilities for the upper and lower laser level, respectively [35]. This has to be transformed to an effective inversion reduction factor $\gamma^{*}=\gamma / f_{a}$ if the effective emission cross section $\sigma_{\text {eff }}$ is used for the rate equations instead of the atomic emission cross section $\sigma=\sigma_{\text {eff }} / f_{a}$ [34]. If the relaxation and thermalization processes are either very slow or very fast with respect to the resonator decay time $\tau_{c}$ or pulse duration $\gamma^{*}$ can be approximated by a constant. Obviously, in this quasi-threelevel system there is no relaxation process of the lower multiplet $\left(\gamma_{b}=0\right)$. Furthermore, the relaxation process of the upper multiplet is very slow with respect to the resonator decay time $\left(1 / \gamma_{a}=\tau_{a} \gg \tau_{c}\right)$. If the thermalization processes within the upper and lower multiplet are fast in comparison to the resonator decay time, $\gamma^{*}=1+f_{b} / f_{a} \approx 1.02$ for the Nd:YGG-laser at $935 \mathrm{~nm}$ at room temperature results. If the thermalization process within the upper multiplet is slow and within the lower multiplet is fast with respect to $\tau_{c}, \gamma^{*}=\left(1+f_{b}\right) / f_{a} \approx 2.19$ occurs. And in the contrary case, where the thermalization process within the upper multiplet is fast and within the lower multiplet slow with respect to $\tau_{c}, \gamma^{*}=1+1 / f_{a} \approx 3.17$ results. Evidently, the extraction efficiency would be dramatically reduced in the two latter cases. 


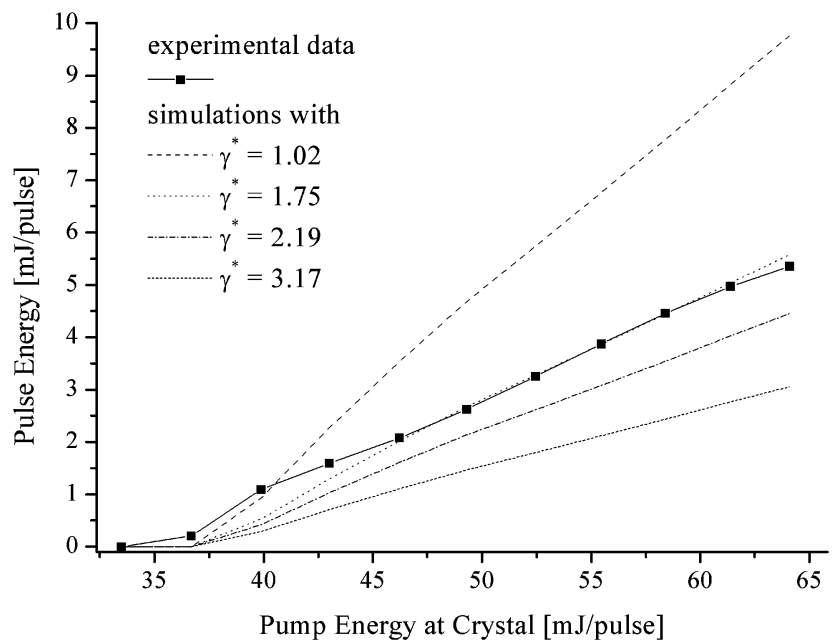

Fig. 13 Experimental and simulated pulse energy data of the oscillator in QS-MM-operation. Simulations account for different inversion reduction factors

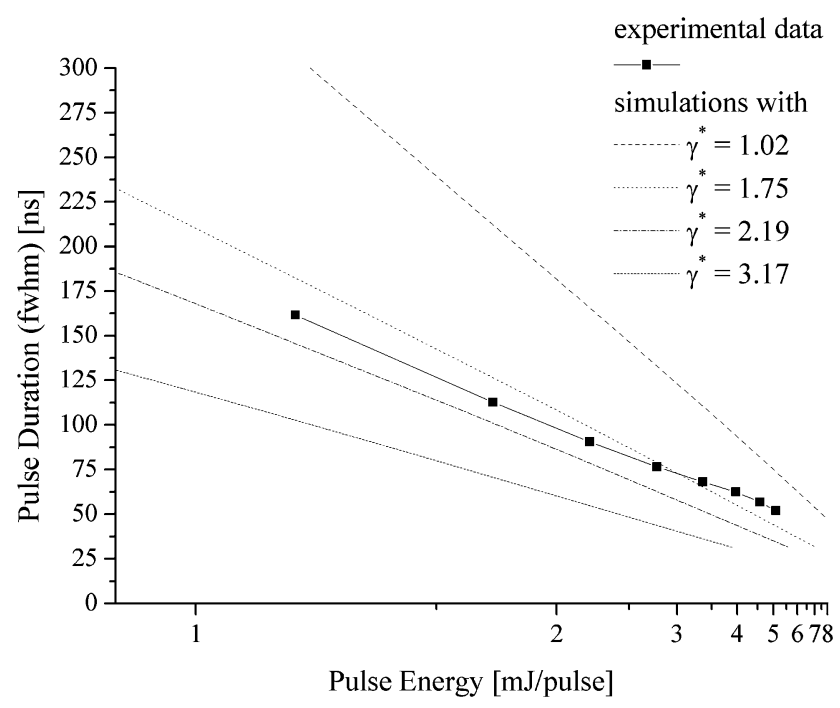

Fig. 14 Comparison of experimental and simulated pulse energy and pulse-duration data of the oscillator in QS-MM-operation. Simulations account for different inversion reduction factors

Calculations of the pulse energy and temporal pulse shape were done for the afore-mentioned effective inversion reduction factors $\gamma^{*}$ and were compared to experimental data. Furthermore, the factor $\gamma^{*}$ was optimized in order to achieve the best agreement between simulation and experimental pulse energy data, which resulted in $\gamma^{*}=1.75$ (see Fig. 13). Also pulse-duration data show good agreement (see Fig. 14) in this case.

It has to be noted that even if that $\gamma^{*}$ value indicates somehow slow thermalization processes, this will have to be proven by more direct measurements. Nevertheless, the important fact is that the extraction efficiency is significantly reduced by some effects. Furthermore, the same reduction of the extraction efficiency was observed in Nd:YGG-based

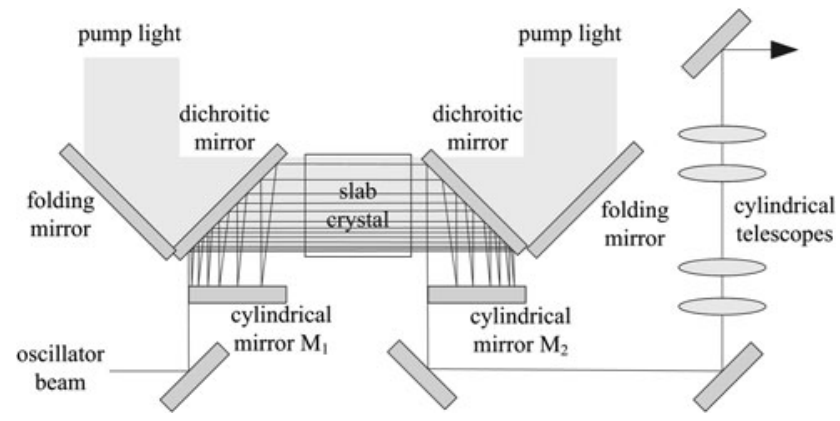

Fig. 15 INNOSLAB-amplifier setup

pulsed single-pass amplifiers. The precise prediction of the amplification properties based on the above measured data is essential for the design of highly efficient oscillators and amplifiers.

\subsection{Amplifier setup}

In order to boost the energy of the Nd:YGG oscillator into the multi-ten-millijoule level, a single INNOSLABamplifier stage was used that is depicted in Fig. 15.

A slab crystal with dimensions of $14.8 \times 15 \times 2 \mathrm{~mm}^{3}$ was used as the active medium. It was made from the same boule as the oscillator crystal. The slab crystal was soldered at both $14.8 \times 15 \mathrm{~mm}^{2}$ faces onto a copper heat sink which was contacted to a water-cooled plate. Both $14.8 \times 2 \mathrm{~mm}^{2}$ faces were antireflex coated for the pump wavelength at $806 \mathrm{~nm}$ as well as at the laser wavelength at $935 \mathrm{~nm}$ and the strongest transition at $1062 \mathrm{~nm}$. The crystal was pumped from both sides through dichroitic mirrors. Their coating, which has identical properties to the pumping mirrors of the oscillator, has a low reflectivity for the pump light and, at $1062 \mathrm{~nm}$, and a high reflectivity at the laser wavelength. At the beginning the oscillator beam passes the sharp edge of the resonator mirror $\mathrm{M}_{1}$ to be coupled into the slab amplifier. Then, the beam is reflected six times from both mirrors $M_{1}$ and $M_{2}$ and travels 13 times through the crystal. Finally, the beam is coupled out geometrically again by passing the sharp edge of mirror $\mathrm{M}_{2}$. Two cylindrical telescopes behind the amplifier are used for beam shaping.

For each crystal side two polarization coupled pump modules were used. Each consisted of 11 passively cooled diodes bars. Stacking of the diodes was done in the fast axis with one-dimensional stacked waveguides with a total front aperture of $11 \times 13 \mathrm{~mm}^{2}$. The setup for one of these pump modules is illustrated in Fig. 16.

The pump beam is homogenized in slow axis behind the polarization coupling by a one-dimensional waveguide. This ensures a homogeneous pump line, even in case of partial emitter failure.

Both pump modules deliver a total pump energy of $828 \mathrm{~mJ}$ within $200 \mu \mathrm{s}$. This value was measured at the position of the slab crystal behind all optics. The cross section 
Fig. 16 Setup of one amplifier pump module. Now, the waveguide stack could be replaced by compact passively cooled vertical diode stacks that are commercially available
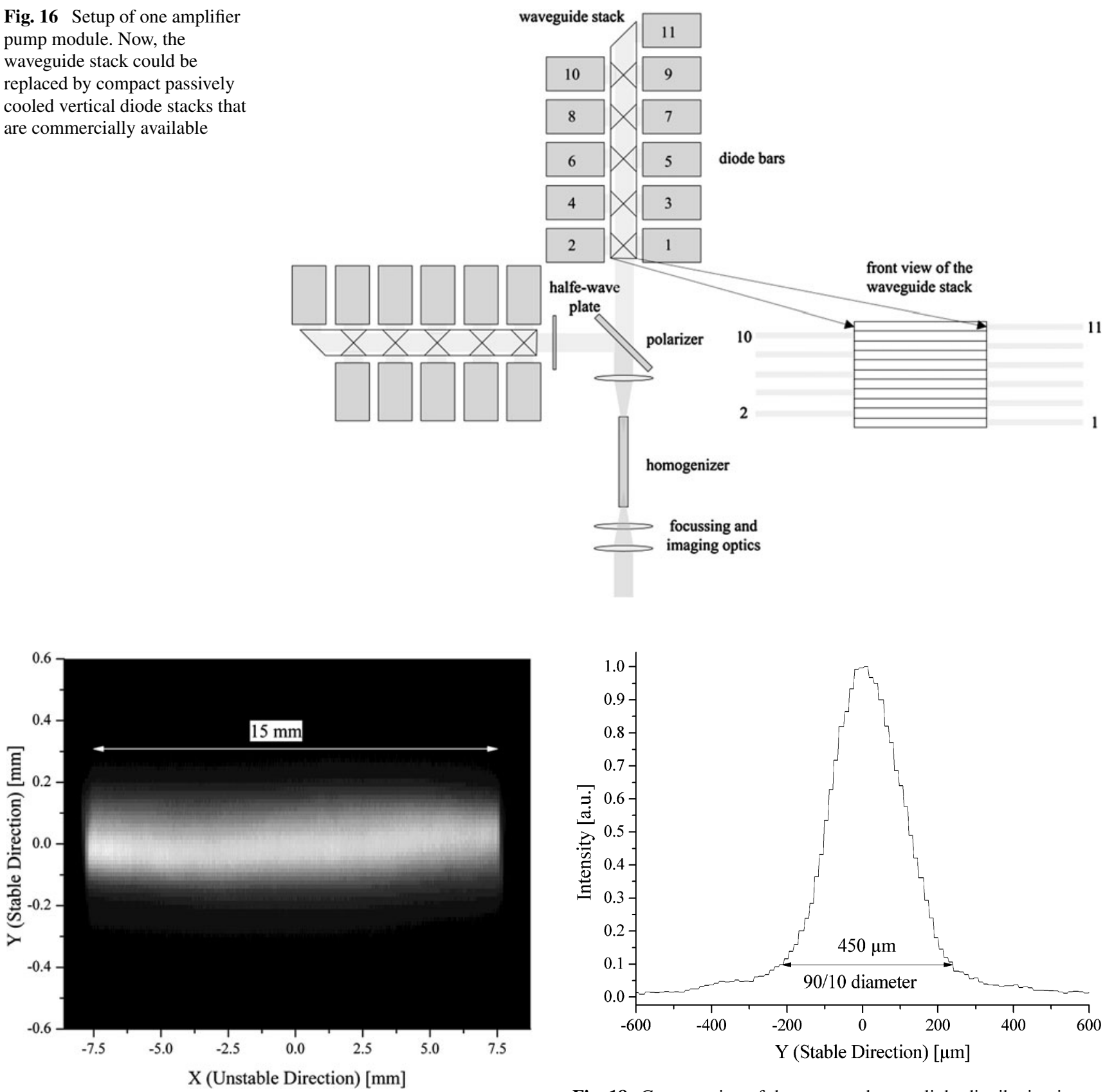

Fig. 18 Cross section of the measured pump light distribution in stable direction at $X=0 \mathrm{~mm}$. The $90 / 10$-diameter of the distribution is measured to be $450 \mu \mathrm{m}$

hind the crystal. In the perpendicular direction the refractive power of the thermal lens is negligible.

A stable-unstable hybrid amplifier configuration was formed with the plane mirror $\mathrm{M}_{1}$ and a plano-convex cylindrical mirror $\mathrm{M}_{2}$. The slab crystal was placed in the center of the 70-mm-long setup. In the unstable direction (parallel to the pump line) the amplifier has a plane-plane configuration. In the stable direction (perpendicular to the pump line) the fundamental amplifier mode is given by the planoconvex mirror arrangement and the cylindrical thermal lens. intersection of these beams was measured to be $225 \mathrm{~mm}$ be- 
Practically, the cylindrical thermal lens is compensated by the plano-convex mirror $\mathrm{M}_{2}$ with a radius of curvature of $448 \mathrm{~mm}$. Furthermore, the fundamental amplifier eigenmode in the stable direction is matched to the height of the pump line in order to achieve high extraction efficiency of the amplifier.

The oscillator beam is consequently matched to the fundamental eigenmode in stable direction. In the unstable direction the beam is expanded by its own divergence given by the plane-plane mirror configuration. Divergence and waist position were adjusted by a cylindrical telescope behind the oscillator. The goal of this expansion in the unstable direction is to match the amplification to result in constant fluences and, therefore, constant amplification properties. Especially in low gain materials like Nd:YGG at $935 \mathrm{~nm}$, it is important to keep the intensity of the beam as high as possible for good extraction efficiencies but below the damage thresholds. Behind the amplifier the beam was strongly astigmatic because of the one-dimensional expansion in the slow axis. By using a second pair of cylindrical telescopes the beam is easily transformed into a round shape.

At the time of devising the amplifier, no passively cooled vertical diode stacks were available. At present, the pump stack could be replaced by commercially available passively cooled stacks with comparable properties. For example JENOPTIK Laser GmbH offers a stack that consists of eight fast-axis collimated diode bar submounts which delivers $808 \mathrm{~nm}$ radiation with a total pulse energy of $216 \mathrm{~mJ}$ (model JOLD-X-QF-8A) at $100 \mathrm{~Hz}$ and $200 \mu$ s pulse duration at an operation current of $165 \mathrm{~A}$. At higher operation currents of about $285 \mathrm{~A}$, which result in higher electrooptical efficiencies, the energy can even be generated within $100 \mu$ s long pulses ${ }^{1}$ also increasing the storage efficiency of the Nd:YGG laser. These kinds of passively cooled stack would significantly decrease volume, mass and complexity of the pump modules and the whole laser transmitter, which is an important issue for a spaceborne DIAL system.

\subsection{Amplification and pulse energy}

The amplification properties were characterized for different pulse energies from the oscillator (Fig. 19) and different pump energies of the pump diodes in the amplifier stage (Fig. 20). In the former case the oscillator beam was attenuated behind the cavity to achieve constant temporal, spatial and spectral beam properties. The oscillator was driven in quasi-continuous wave mode (QCW), in QS-MM-operation and QS-SLM-operation, seeded at the gain maximum at $935.31 \mathrm{~nm}$ in Nd:YGG. The amplifier shows nearly the same performance in QS-MM- and QS-SLM-operation. Because of the better performance of the oscillator in QS-MM, the

\footnotetext{
${ }^{1}$ Online product information, www.jenoptic.com (2010).
}

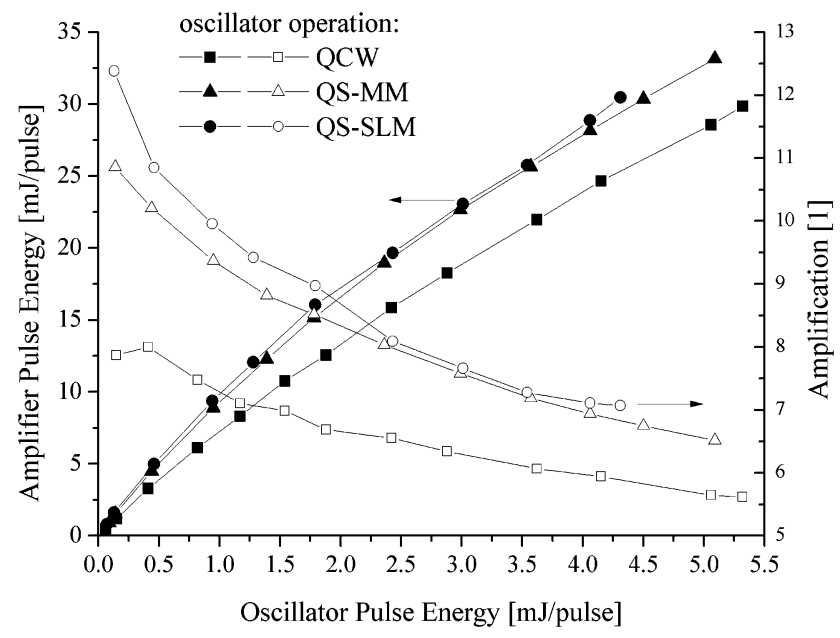

Fig. 19 Pulse energy and amplification for different pulse energies of the oscillator and operation modes

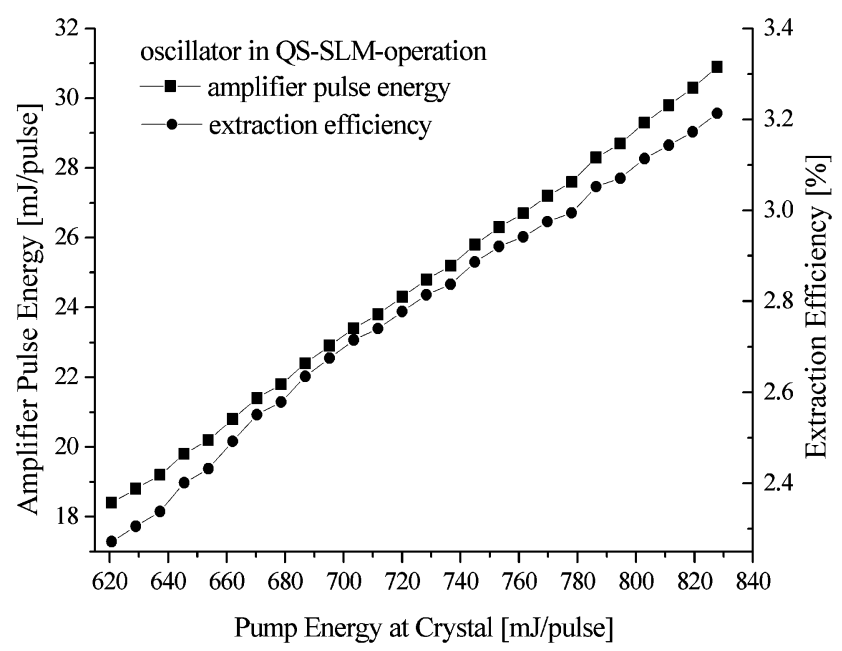

Fig. 20 Pulse energy and extraction efficiency of the amplifier versus the pump energy of the amplifier stage at the crystal

beam could be amplified to $33.2 \mathrm{~mJ}$. In QS-SLM-operation $30.5 \mathrm{~mJ}$ could be generated. In QCW-operation $28.9 \mathrm{~mJ}$ were reached. This corresponds to a gain of 6.5 for the QSMM-operation, 7.1 for QS-SLM-operation and 5.6 for the QCW-operation. It has to be noted that in QCW- and in QSMM-operation, the oscillator emits at $935 \mathrm{~nm}$ and $938 \mathrm{~nm}$ simultaneously.

The extraction efficiency at maximum pump level was $3.2 \%$ in single-frequency mode. Both pulse energy behind the amplifier and extraction efficiency increased with higher pump energies. Thus, higher efficiencies are in principle possible using this setup at higher pump levels. This might be limited at a certain point by parasitic lasing around $1062 \mathrm{~nm}$, the gain maximum of Nd:YGG.

Furthermore, the amplifier is not yet fully optimized concerning the overlap of the laser beam and pump volume. The main reason for this is that the amplifier design had to be 


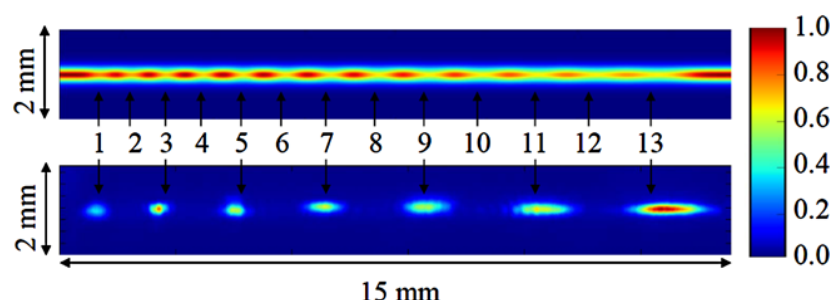

$15 \mathrm{~mm}$

Fig. 21 Top: Calculated distribution of the stored energy after the amplification process; bottom: measurements of the intensity distribution of the amplifier beam for odd pass numbers

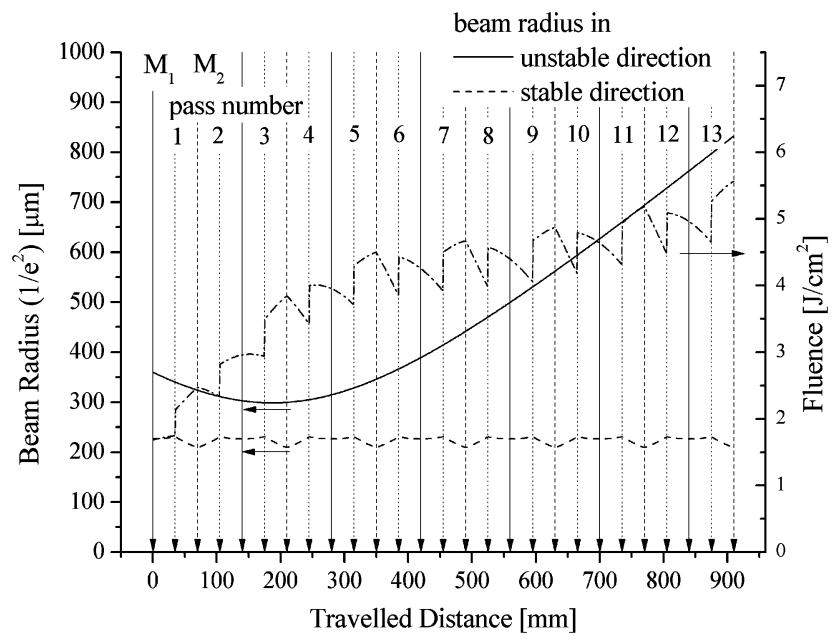

Fig. 22 Calculated beam caustics in stable and unstable direction as well as the appropriate mean energy fluence for the propagation of the laser beam inside the whole amplifier. At distance $0 \mathrm{~mm}$ the beam passes the mirror $M_{1}$, after $910 \mathrm{~mm}$ of traveled distance the beam passes the mirror $M_{2}$ and is coupled out

based on preliminary crystal data not allowing for precise predictions of the oscillator performance and amplification properties. Only for the last amplifier passes do high-energy fluences of the laser beam lead to high extraction efficiencies. This is shown by both calculated energy distribution in the slab crystal after the pulse extraction and measured intensity distribution of the laser beam for odd pass numbers (see Fig. 21) as well as in the calculated beam caustics and appropriate energy fluences (see Fig. 22). Here, the mean energy fluence

$F=\frac{E_{p}}{\pi \omega_{\text {stable }} \omega_{\text {unstable }}}$

was calculated from the pulse energy $E_{p}$ which increases with the crystal passes and the $1 / e^{2}$ beam radii $\omega_{\text {stable }}$ and $\omega_{\text {unstable }}$ in stable and unstable direction, respectively. The peak value of the energy fluence is again a factor of two higher than the mean value. The pulse energy $E_{p}$ after each amplification step in the slab crystal was calculated making use of the theory introduced by Frantz and Nodvik [36]. The distribution of the extractable energy density in the laser crystal was scaled with a scalar calibration factor in order to obtain realistic gain values that lead to the measured total output pulse energy of $30.5 \mathrm{~mJ}$ behind the amplifier out of $4.3 \mathrm{~mJ}$ input pulse energy.

While in vertical (stable) direction the laser beam and the pump volume are perfectly matched, in horizontal (unstable) direction the broadening cannot perfectly be matched to the amplification. This is mainly caused by the low amplification in Nd:YGG at $935 \mathrm{~nm}$ being smaller than the broadening of the beam in the horizontal (unstable) direction caused by diffraction. The mean energy fluence increases for the first crystal passes from about $2 \mathrm{~J} / \mathrm{cm}^{2}$ to about $5 \mathrm{~J} / \mathrm{cm}^{2}$ for the last passes, which is well below the saturation fluence. Furthermore, the energy fluence inside the laser medium is about a factor of eight for the first passes and of five for the last passes lower than in the resonator, which is the main reason for the lower extraction efficiencies. It has to be noted that an amplifier is principally not inferior to an oscillator concerning its extraction efficiency. Significantly higher efficiencies are expected at higher energy densities.

There are different ways how the performance could be optimized. Firstly, higher pumping levels would increase the amplification compared to the broadening in unstable direction. Secondly, a shorter distance between mirrors $\mathrm{M}_{1}$ and $\mathrm{M}_{2}$ would reduce the broadening of the traveling beam compared to its amplification. The latter can only be implemented if the beam path is folded in stable direction which was not set up here. Thirdly, the incoming beam can be preamplified in order to reduce its divergence angle at a constant fluence. Consequently, higher extraction efficiencies are expected in an identical second amplifier step.

\subsection{Beam quality}

The beam-quality was measured behind the final pair of cylindrical telescopes. The beam has a Gaussian shape only in the far field. In the near field diffraction structures appear which might be generated by thermal gradients in the slab crystal in stable direction (see Fig. 23).

Even with these diffraction structures which are typical for the slab crystal geometry a beam propagation factor as low as $\mathrm{M}^{2}<1.4$ has been achieved. This fully meets the beam-quality requirements for a $\mathrm{H}_{2} \mathrm{O}$ lidar transmitter.

\subsection{Spectral properties and tunability}

In order to measure both stability of the center frequency and spectral width from pulse to pulse in single-longitudinalmode operation heterodyne measurements were performed (see Fig. 24).

The measurement of 579 beat signals yields a peak-topeak frequency fluctuation of better than $\pm 8.5 \mathrm{MHz}$ and a rms fluctuation of $2.9 \mathrm{MHz}$, respectively. The linewidth was 
Fig. 23 Measurements of the amplifier beam diameters at different positions (beam caustic) behind the last pair of cylindrical telescopes at the maximum pulse energy. At eight positions the normalized intensity distribution is also given. All images have equal dimensions

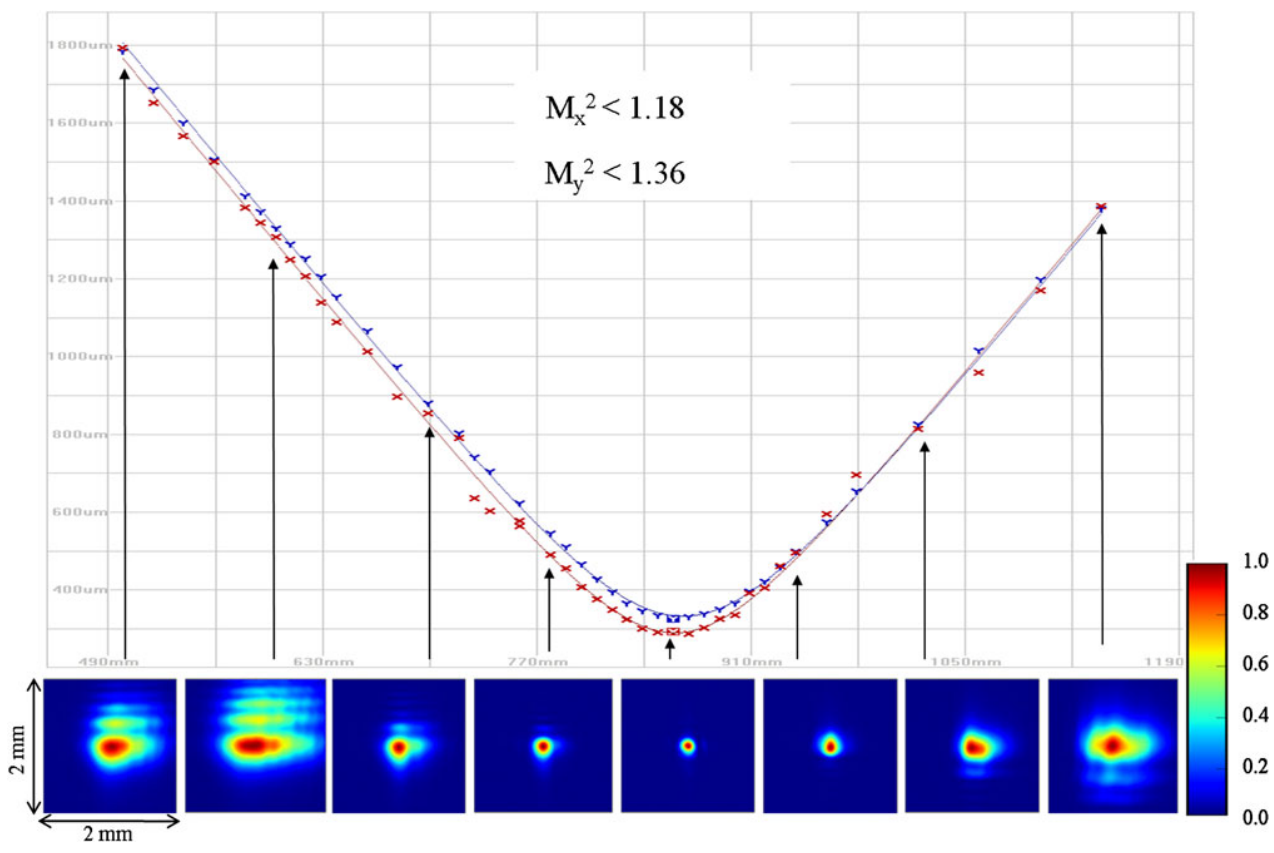

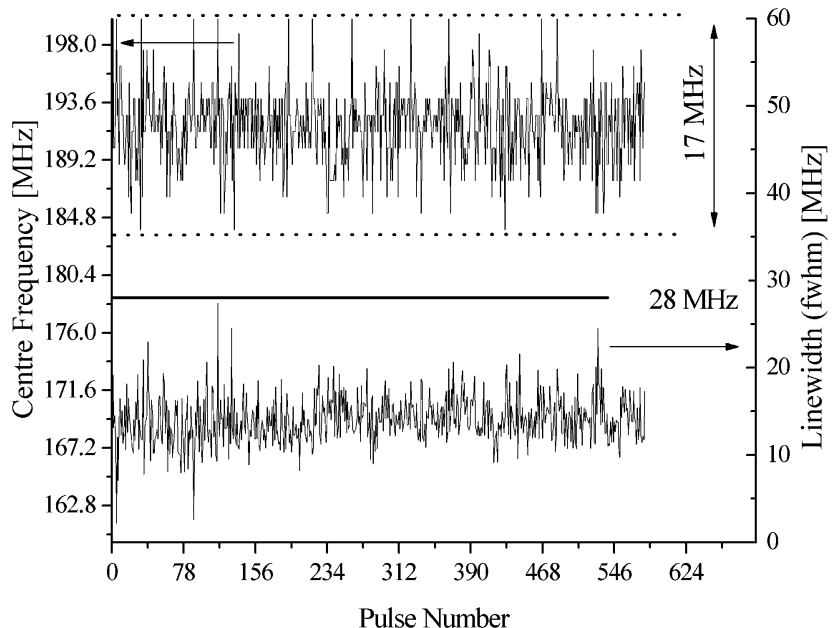

Fig. 24 Heterodyne measurements of the center frequency and linewidth of the Nd:YGG laser tuned to the maximal single-frequency pulse energy at $935.31 \mathrm{~nm}$

measured to be $14.0 \pm 2.7 \mathrm{MHz}$ and its maximum was lower than $28 \mathrm{MHz}$. Following the pulse duration of $52.2 \mathrm{~ns}$, the time-bandwidth product is 0.731 . Both frequency stability and linewidth fully meet the requirements for a spaceborne water-vapor lidar transmitter [9].

The concept for a spaceborne lidar as proposed for the WALES instrument suggests the use of four wavelengths covering a spectral range of $0.345 \mathrm{~nm}$ with its center at $935.7335 \mathrm{~nm}$ (vac.). Since the emission profile of the $\mathrm{R}_{2}-\mathrm{Z}_{5}$ transition is not particularly broad, the question remains over what wavelength range single-longitudinal-mode operation can be maintained by injection seeding.

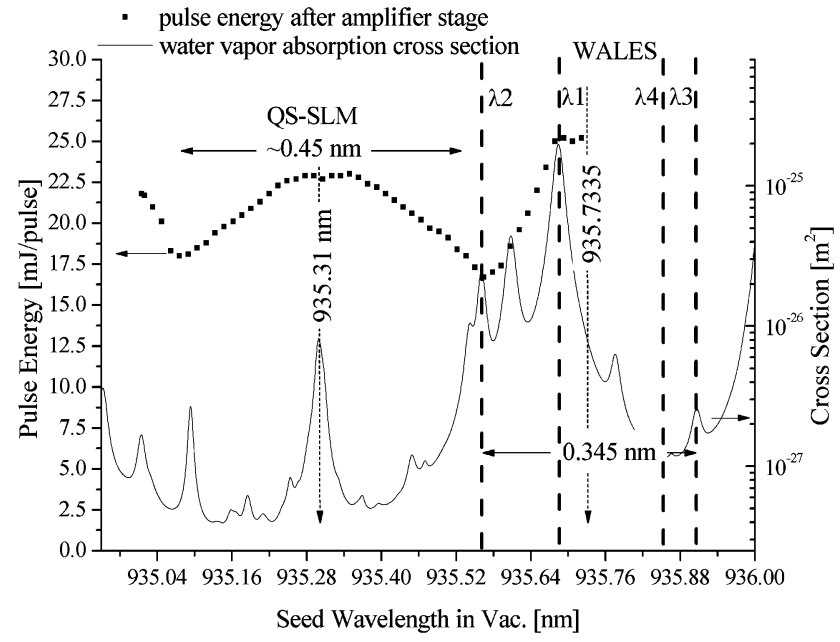

Fig. 25 Tunability of the Nd:YGG laser in single-frequency operation mode and relevant water-vapor absorption cross sections [38]

Tuning the Nd:YGG laser into the flanks of the emission spectrum directly leads to a gain reduction. If the seed wavelength is detuned from the gain maximum beyond a certain level, the seed power is not sufficient to suppress competing modes with higher gain. Even modes at $938 \mathrm{~nm}$ of the weaker $\mathrm{R}_{1}-\mathrm{Z}_{5}$ transition might occur. To answer this question the DFB-diode seed laser was tuned from $935.01 \mathrm{~nm}$ to $935.72 \mathrm{~nm}$ at a seed power of about $5 \mathrm{~mW}$. In order to distinguish between successful and unsuccessful seeding the temporal behavior and energy was analyzed. If the seed spectrum is tuned away from the gain maximum, a reduction of the pulse energy and an increase of the pulse build-up time occur. At the transition to multimode operation both pulse power and build-up time become unstable. This is the case 
Table 5 Summary of laser properties

\begin{tabular}{lll}
\hline Parameter group & Parameter & Value \\
\hline Spectral & Center wavelength (gain peak) & $935.31 \mathrm{~nm}$ (vac.) \\
& Tuning range (SLM) & $935.10 \mathrm{~nm}-935.55 \mathrm{~nm}$ (vac.) \\
& Width & $<28 \mathrm{MHz}$ (peak), 14.0 $\pm 2.7 \mathrm{MHz}$ \\
& Stability of center wavelength & $< \pm 8.5 \mathrm{MHz}$ (p-t-p), 2.9 MHz (rms) \\
& Purity & $>99.996 \%[39]$ \\
Energetic & cw seed power & $\sim 5 \mathrm{~mW}$ \\
& Pulse energy oscillator & $4.3 \mathrm{~mJ}$ \\
& Pulse energy amplifier & $30.5 \mathrm{~mJ}$ \\
& Efficiency oscillator & $5.7 \%$ (pump light at crystal to laser out) \\
& Efficiency amplifier & $3.2 \%$ (pump light at crystal to laser out) \\
& Efficiency MOPA & $3.3 \%$ (pump light at crystals to laser out) \\
Temporal & Pulse-duration MOPA & $52.5 \mathrm{~ns}$ \\
& Repetition rate & $100 \mathrm{~Hz}$ \\
Spatial & Beam-quality MOPA & $M^{2}<1.4$ \\
\hline
\end{tabular}

when, besides the seeded mode, at least one more longitudinal mode with comparable intensity occurs. With respect to this criterion for SLM, the laser source could be tuned over a range of about $0.45 \mathrm{~nm}(935.10-935.55 \mathrm{~nm})$. In Fig. 25 the pulse energy of the MOPA system is plotted versus the seed wavelength. Overlaid are the water-vapor absorption cross section (from HITRAN 2009 [38]) and the proposed WALES wavelength $\lambda_{1}$ to $\lambda_{4}$.

A more relevant criterion concerning the spectral properties which make a laser suitable for $\mathrm{H}_{2} \mathrm{O}$ DIAL measurements is the spectral purity. For the WALES instruments a spectral purity of $99.9 \%$ is required. Since those values cannot be measured with sufficient accuracy using common spectrometers, long-pass absorption measurements have been performed.

A spectral purity of more than $99.996 \%$ was measured. Details of these experiments, together with first water-vapor profile measurements, are published in a companion paper [39].

Concerning the prospects for a spaceborne DIAL instrument, the main drawback of the Nd:YGG laser is the limitation of the tuning range which does not exactly match the proposed WALES wavelengths. Addressing different wavelengths between $935.3 \mathrm{~nm}$ and $936.6 \mathrm{~nm}$ with compositionally tuned $\mathrm{Nd}: \mathrm{Y}_{x} \mathrm{Lu}_{3-x} \mathrm{Ga}_{5} \mathrm{O}_{12}$-crystals (Nd:YLuGG) with $(0 \leq x \leq 3)$ is currently under investigation. This is based on measurements of the emission spectrum of pure Nd:YGG crystals as well as Nd:LuGG crystals [13]. Preliminary experiments with a Q-switched laser oscillator using different Nd:YLuGG crystals show that the WALES wavelengths can be generated at efficiencies comparable to Nd:YGG at $935 \mathrm{~nm}$. Details about these measurements will be published elsewhere.

\subsection{Overview of laser properties and comparison}

In conclusion we have developed a laser whose properties fulfill the requirements for a spaceborne water-vapor DIAL transmitter according to the WALES concept except its water-vapor line center wavelengths. Table 5 gives an overview of the achieved performance.

The efficiency of the Nd:YGG MOPA is comparable to current OPO or Ti:Sapphire based systems. In order to determine the state-of-the-art conversion efficiency from the diode pump light at $808 \mathrm{~nm}$ (at the $\mathrm{Nd}$ :YAG crystal) to $935 \mathrm{~nm}$ at the relevant working point (QS-SLM, $\sim 100 \mathrm{~Hz}$, ns-pulses, comparable pulse energies), we compared the conversion efficiencies of the different converting steps separately, taking into account data of three relevant published laser sources (see Table 6).

A high performance airborne water-vapor DIAL system at $935 \mathrm{~nm}$ was developed by DLR. This system delivered $45 \mathrm{~mJ}$ pulses at $100 \mathrm{~Hz}$ repetition rate and adequate spectral purity based on OPO technology [40]. The conversion efficiency of the OPO was $29 \%$. Today's highly efficient singlefrequency pump sources at $1064 \mathrm{~nm}$ have an efficiency of about $24 \%$ (pump light at $808 \mathrm{~nm}$ at the laser crystal to $1064 \mathrm{~nm}$ ) [29]. The efficiency of the second harmonic generation depends strongly on the peak intensity of the incident beam and is, therefore, limited by damage thresholds in this regime. Conversion efficiencies of $55 \%$ are typical for the second harmonic generation (see [40]). The overall conversion efficiency (808 $\mathrm{nm}$ to $935 \mathrm{~nm}$ ) in OPO-based converters is currently limited to about $3.9 \%$. A further shortcoming of OPO and Ti:Sapphire-based laser systems, comprising of two frequency converter steps, is their high complexity in comparison to direct pumped solid-state lasers. This usually increases the probability of failure, which is unacceptable for space missions. 
Table 6 Efficiency of comparable laser sources at $935 \mathrm{~nm}$ based on Ti:Sapphire and OPO technology

\begin{tabular}{llll}
\hline Conversion & Efficiencies & & \\
\hline & {$[29]$} & {$[40]$} & {$[41]$} \\
$808 \mathrm{~nm}$ (at laser crystal) to $1064 \mathrm{~nm}$ & $\mathbf{2 4 . 7 \%}$ & $13.8 \%$ & - \\
$1064 \mathrm{~nm}$ to $532 \mathrm{~nm}$ & - & $\mathbf{5 5 . 0 \%}$ & - \\
$532 \mathrm{~nm}$ to $935 \mathrm{~nm}(\mathrm{Ti}:$ Sapphire) & - & $\mathbf{2 9 \%}$ & - \\
$532 \mathrm{~nm}$ to $935 \mathrm{~nm}(\mathrm{OPO})$ & - & Calculated maximal efficiencies & \\
& & $13.6 \%$ & $\mathbf{3 . 0 \%}$ \\
$808 \mathrm{~nm}$ to $532 \mathrm{~nm}$ & & $\mathbf{3 . 9 \%}$ & \\
$808 \mathrm{~nm}$ to $935 \mathrm{~nm}$ (Ti:Sapphire) & &
\end{tabular}

\section{Summary}

To summarize, a Nd:YGG laser has been specifically designed to meet the stringent requirements for water-vapor differential absorption lidar measurements from air and space.

Since current transmitters for $\mathrm{H}_{2} \mathrm{O}$ DIAL such as Ti:Sapphire lasers or OPOs generally require an additional (efficiency-eating) frequency conversion stage to generate radiation in the 935-nm band of water vapor, the goal of this work was to demonstrate that a diode-pumped laser material is not only able to directly generate the required wavelengths but, at the same time, also delivers energies in the range required for airborne or spaceborne applications together with excellent spatial and spectral properties.

The Nd:YGG-laser system is set up as a master-oscillator power-amplifier configuration. Since Nd:YGG is a poorly investigated material, additional important parameters such as fluorescence spectrum or thermo-optical properties to optimize the performance under high pump power had to be measured prior to the design of the oscillator. For a better understanding of the limitations and design issues of $\mathrm{Nd}$ :YGG-based lasers, we summarized the relevant crystal properties in comparison to the well-known Nd:YAG crystal. The roundtrip losses of the cavity as well as the total coupling efficiency were measured. Calculations of both pulse energy and temporal pulse shape that were based on these data show good agreement with experimental results. This model allows for the prediction of energetic and temporal pulse properties of Nd:YGG-based oscillators.

At an energy of $85 \mathrm{~mJ}$ from the fiber-coupled pump diodes, the laser generated $7.7 \mathrm{~mJ}$ in Q-switched operation with a slope efficiency of $15.2 \%$ and a pulse duration of 52.5 ns. Single-frequency operation was obtained by injecting light from a DFB-diode laser. For this purpose, two quarter waveplates were added to the linear laser resonator which was stabilized to the seed wavelength using the ramp-andfire technique. The beam quality is excellent. An $\mathrm{M}^{2}$-value of 1.06 was experimentally determined.
In order to boost the pulse energy into the multi-tenmillijoule range, an amplifier stage according to the INNOSLAB concept was chosen. The thin slab crystal $(14.8 \times$ $15 \times 2 \mathrm{~mm}^{3}$ ) was end pumped from both ends by a total of 44 laser diode bars through a free-space transfer optic.

At a maximum diode pump energy at the crystal of $828 \mathrm{~mJ}$ within $200 \mu \mathrm{s}$ and 13 passes of the oscillator beam through the slab crystal, the amplifier generated $28.9 \mathrm{~mJ}$ of energy in QCW mode and $33.2 \mathrm{~mJ}$ in Q-switched mode, respectively. When Q-switched and injection seeded at the gain center, the system generated $30.5 \mathrm{~mJ}$ in singlefrequency operation. Due to the low energy fluence of $2-$ $5 \mathrm{~J} / \mathrm{cm}^{2}$, the optical efficiency of the amplifier is limited to $3.2 \%$. Higher efficiencies are expected at higher energy fluences. The potential of Nd:YGG at $935 \mathrm{~nm}$ concerning efficiency is impressively demonstrated by the oscillator. Here, an efficiency of 5.7\% in QS-SLM-operation and 9.3\% in QS-MM-operation without intracavity quarter-wave plates are achieved at energy fluence at the laser medium of $13 \mathrm{~J} / \mathrm{cm}^{2}$ and $23 \mathrm{~J} / \mathrm{cm}^{2}$, respectively. Principally, these efficiencies are also possible in an amplifier stage at these fluences. Consequently, further enhancement of the amplifier output energy is straightforward by using another subsequent amplifier stage of identical design. It is expected that the degradation in beam quality caused by that amplifier stage will be in the range of the first one. But this will have to be investigated.

By tuning the seed wavelength across the gain profile, it was found that the range of single-frequency operation extended over $0.45 \mathrm{~nm}$ from $935.10 \mathrm{~nm}$ to $935.55 \mathrm{~nm}$. Beyond this range the lasers tended to show parasitic oscillation at $938 \mathrm{~nm}$.

The spatial and spectral properties of this laser system exceed the requirements for a transmitter for spaceborne water-vapor DIAL by far. The $\mathbf{M}^{2}$-value was measured to be less than 1.4 , the bandwidth was $14.0 \pm 2.7 \mathrm{MHz}$ with its maximum below $28 \mathrm{MHz}$. The frequency stability relative to the seed laser frequency was better than $\pm 8.5 \mathrm{MHz}$ (pk-pk) and 2.9 MHz (rms), respectively. The spectral purity was also excellent, but these results, together with first 
lidar measurement, are the subject of a companion publication [39].

The only drawback of the current system is that singlefrequency operation was not possible at the strongest watervapor absorption lines at $935.685 \mathrm{~nm}$ or $935.561 \mathrm{~nm}$ in the 935-nm band, lines that are suitable to measure low tropospheric and stratospheric water-vapor concentrations. There is a high probability that $\mathrm{Nd}: \mathrm{Y}_{x} \mathrm{Lu}_{3-x} \mathrm{Ga}_{5} \mathrm{O}_{12}$ crystals with an optimized composition $(0 \leq x \leq 3)$ will close this gap.

The system described here is a major advancement to increase the technology readiness level of directly pumped solid-state lasers as transmitters for water-vapor DIAL systems in space. This first attempt already comes up to the optical efficiencies and exceeds the spectral quality achieved by competing systems such as OPOs or Ti:Sapphire lasers and - with further optimization-thus shows much promise for transmitters in both airborne and spaceborne watervapor DIAL systems where high efficiencies are a prerequisite for mission feasibility.

Acknowledgements This project was funded by DLR space agency in name of the BMWi under contract number FKZ 50 EE 0714. We would like to thank Prof. Wolfgang Heddrich, Peter Becker and Alexander Wissel for their assistance in developing the laser source and in the lab, Dieter Plum and Carsten Heuer for the mechanical constructions, Martin Traub for the design of the amplifier pump optics and Bernd Jungbluth for helpful discussions. For the laser crystal material and support we also acknowledge FEE-GmbH in Idar-Oberstein and the Institute for Laser Physics (ILP) of the University of Hamburg.

Open Access This article is distributed under the terms of the Creative Commons Attribution Noncommercial License which permits any noncommercial use, distribution, and reproduction in any medium, provided the original author(s) and source are credited.

\section{References}

1. N.P. Barnes, Opt. Mat. 27, 1653 (2005)

2. R.M. Schotland, in Proc. Fourth Symp. Remote Sens. Environ., Ann Arbor, Michigan (ERIM, Michigan, 1966), p. 273

3. S. Solomon, D. Qin, M. Manning, Z. Chen, M. Marquis, K.B. Averyt, M. Tignor, H.L. Miller (eds.), Climate Change 2007: The Physical Science Basis. Contribution of Working Group I to the Forth Assessment Report of the Intergovernmental Panel on Climate Change (Cambridge University Press, Cambridge, 2007)

4. É. Gérard, D.G.H. Tan, L. Garand, V. Wulfmeyer, G. Ehret, P. Di Girolamo, Bull. Am. Meteorol. Soc. 85, 237 (2004)

5. E.V. Browell, S. Ismail, W.B. Grant, Appl. Phys. B 67, 399 (1998)

6. A. Helière, J.-L. Bézy, P. Bensi, P. Ingmann, in Proc. SPIE Int. Soc. Opt. Eng., vol. 4881 (2003), p. 24

7. P. Di Girolamo, D. Summa, H. Bauer, V. Wulfmeyer, A. Behrendt, G. Ehret, B. Mayer, M. Wirth, C. Kiemle, ESA SP 561, 957 (2004)

8. P. Di Girolamo, A. Behrendt, C. Kiemle, V. Wulfmeyer, H. Bauer, D. Summa, A. Dörnbrack, G. Ehret, Remote Sens. Environ. 112, $1552(2008)$
9. ESA, Report for assessment: WALES-water vapour lidar experiment in space, ESA SP 1257 (2) (2001)

10. B.M. Walsh, N.P. Barnes, R.L. Hutcheson, R.W. Equall, B. Di Bartolo, J. Opt. Soc. Am. B 15, 2794 (1998)

11. N.P. Barnes, B.M. Walsh, R.L. Hutcheson, Adv. Solid State Laser 1, 522 (1996)

12. B.M. Walsh, N.P. Barnes, R.L. Hutcheson, R.W. Equall, IEEE J. Quantum Electron. 37, 1203 (2001)

13. R. Treichel, C. Czeranowsky, B. Ileri, K. Petermann, G. Huber, Eur. Space Agency, ESA SP 554, 639 (2004)

14. S.G.P. Strohmaier, H.J. Eichler, C. Czeranowsky, B. Ileri, K. Petermann, G. Huber, Opt. Commun. 275, 170 (2007)

15. F. Kallmeyer, M. Dziedzina, X. Wang, H.J. Eichler, C. Czeranowsky, B. Ileri, K. Petermann, G. Huber, Appl. Phys. B 89, 305 (2007)

16. Z. Lin, X. Wang, F. Kallmeyer, H.J. Eichler, C. Gao, Opt. Express 18, $6131(2010)$

17. T.J. Axenson, N.P. Barnes, D.J. Reichle, E.E. Köhler, J. Opt. Soc. Am. B 19, 1535 (2002)

18. Q. Li, B. Feng, Z. Wei, D. Zhang, D. Li, Z. Zhang, H. Zhang, J. Wang, Opt. Lett. 33, 261 (2008)

19. Q. Li, B. Feng, D. Zhang, Z. Zhang, H. Zhang, J. Wang, Appl. Opt. 48, 1898 (2009)

20. P. Russbueldt, T. Mans, G. Rotarius, J. Weitenberg, H.D. Hoffmann, R. Poprawe, Opt. Express. 17, 12230 (2009)

21. J. Löhring, A. Meissner, V. Morasch, P. Becker, W. Heddrich, D. Hoffmann, Proc. SPIE 7193, 71931 Y (2009)

22. G.A. Slack, D.W. Oliver, Phys. Rev. B 5, 3370 (1972)

23. P.A. Giesting, A.M. Hofmeister, Phys. Rev. B 65, 144305 (2002)

24. K. Petermann, Private communication (2006)

25. R. Penndorf, J. Opt. Soc. Am. 47, 176 (1957)

26. A.A. Kaminskii, Laser Crystals (Springer, Berlin/Heidelberg, 1990)

27. J. Löhring, K. Nicklaus, N. Kujath, D. Hoffmann, Proc. SPIE 6451, 64510I (2007)

28. W. Koechner, Solid-State Laser Engineering (Springer, Berlin/Heidelberg, 2006)

29. J. Luttmann, K. Nicklaus, V. Morasch, S. Fu, M. Höfer, M. Traub, H.-D. Hoffmann, R. Treichel, C. Wührer, P. Zeller, Proc. SPIE 6871, 687109 (2008)

30. V. Magni, Appl. Opt. 25, 107 (1986)

31. V. Evtuhov, A.E. Siegman, Appl. Opt. 4, 142 (1965)

32. S.W. Henderson, E.H. Yuen, E.S. Fry, Opt. Lett. 11, 715 (1986)

33. K. Nicklaus, V. Morasch, M. Höfer, J. Luttmann, M. Vierkötter, M. Ostermeyer, J. Höffner, Proc. SPIE 6451, 64511L (2007)

34. J.J. Degnan, IEEE J. Quantum Electron. 25, 214 (1989)

35. J.J. Degnan, D.B. Coyle, R.B. Kay, IEEE J. Quantum Electron. 34, 887 (1998)

36. L.M. Frantz, J.S. Nodvik, Appl. Phys. 34, 2346 (1963)

37. D. Findlay, R.A. Clay, Phys. Lett. 20, 277 (1966)

38. L.S. Rothman, I.E. Gordon, A. Barbe, D.C. Benner, P.F. Bernath, M. Birk, L.R. Brown, V. Boudon, J.-P. Champion, K.V. Chance, L.H. Coudert, V. Dana, M.V. Devi, S. Fally, J.-M. Flaud, R.R. Gamache, A. Goldman, D. Jacquemart, N. Lacome, J.-Y. Mandin, S.T. Massie, S. Mikhailenko, A. Nikitin, J. Orphal, V. Perevalov, A. Perrin, C.P. Rinsland, M. Šimečková, M.A.H. Smith, S. Tashkun, J. Tennyson, R.A. Toth, A.C. Vandaele, J. Van der Auwera, J. Quant. Spectrosc. Radiat. Transf. 110, 533 (2009)

39. A. Fix, G. Ehret, J. Löhring, D. Hoffmann, M. Alpers, Appl. Phys. B (2010). doi: 10.1007/s00340-010-4310-5

40. M. Wirth, A. Fix, P. Mahnke, H. Schwarzer, F. Schrandt, G. Ehret, Appl. Phys. B 96, 201 (2009)

41. F. Kallmeyer, A. Hermerschmidt, H.J. Eichler, H.H. Klingenberg, S. Nikolov, IEEE ASSP Mag. 98, 718 (2005) 